\title{
TRIANGULAR DECOMPOSITION OF SKEIN ALGEBRAS
}

\author{
THANG T. Q. LE
}

\begin{abstract}
By introducing a finer version of the Kauffman bracket skein algebra, we show how to decompose the Kauffman bracket skein algebra of a surface into elementary blocks corresponding to the triangles in an ideal triangulation of the surface. The new skein algebra of an ideal triangle has a simple presentation. This gives an easy proof of the existence of the quantum trace map of Bonahon and Wong. We also explain the relation between our skein algebra and the one defined by Muller, and use it to show that the quantum trace map can be extended to the Muller skein algebra.
\end{abstract}

\section{INTRODUCTION}

1.1. Kauffman bracket skein algebra of surface. Throughout this paper $\mathcal{R}$ is a commutative ring with unit 1 and a distinguished invertible element $q^{1 / 2} \in \mathcal{R}$.

Suppose $\mathfrak{S}=\overline{\mathfrak{S}} \backslash \mathcal{P}$, where $\overline{\mathfrak{S}}$ is a compact oriented 2-dimensional manifold with (possibly empty) boundary $\partial \overline{\mathfrak{S}}$ and $\mathcal{P}$ is a finite set. The Kauffman bracket skein algebra $\dot{\mathscr{S}}(\mathfrak{S})$, introduced by Przytycki $[\mathrm{Pr}$ and Turaev [Tu], is defined as the $\mathcal{R}$-module spanned by isotopy classes of framed unoriented links in $\mathfrak{S} \times(0,1)$ modulo the skein relation (11) and the trivial loop relation (2):

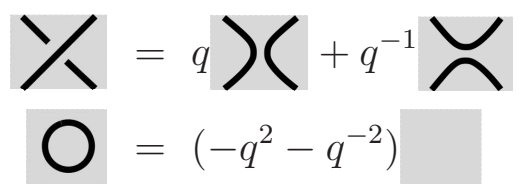

For a detailed explanation of these formulas, as well as other formulas and notions in the introduction, see Section 2. There is a natural product making $\mathscr{\mathscr { S }}(\mathfrak{S})$ an $\mathcal{R}$-algebra, which has played an important role in low-dimensional topology and quantum topology. In particular, it is known that $\mathscr{\mathscr { S }}(\mathfrak{S})$ is a quantization of the $S L_{2}(\mathbb{C})$-character variety of the fundamental group of $\mathfrak{S}$ along the Weil-Petersson-Goldman bracket [Tu, Bul, [PS1, BFK]. The algebra $\mathscr{S}(\mathfrak{S})$ and its cousin defined for 3-manifolds have helped to establish the AJ conjecture, relating the Jones polynomial and the A-polynomial of a knot, for a certain class of knots [Le, LZ]. A construction of Topological Quantum Field Theory is based on $\mathscr{S}(\mathfrak{S})$ [BHMV]. Recently, $\mathscr{\mathscr { S }}(\mathfrak{S})$ is found to have relations with quantum cluster algebras and quantum Teichmüller spaces [BW1, and we also discuss these relations in this paper.

Date: July 21, 2018.

Supported in part by National Science Foundation. 2010 Mathematics Classification: Primary 57N10. Secondary 57M25.

Key words and phrases: Kauffman bracket skein module, Chebyshev homomorphism. 
The skein algebra $\dot{\mathscr{S}}(\mathfrak{S})$ is defined using geometric objects in a 3 -manifold, and we want to understand its algebraic aspects.

1.2. Decomposition. Assume that each connected component of the boundary $\partial \mathfrak{S}$ is diffeomorphic to the open interval $(0,1)$. Such a $\mathfrak{S}$ is called a punctured bordered surface in this paper. Every connected component of $\partial \mathfrak{S}$ is called a boundary edge of $\mathfrak{S}$.

Very often $\mathfrak{S}$ has an ideal triangulation. This means, $\mathfrak{S}$ can be obtained from a finite collection of disjoint ideal triangles by gluing together some pairs of edges of these triangles. Here an ideal triangle is a triangle without vertices. We want to know if one can build, or understand, the skein algebra of $\mathfrak{S}$ from those of the ideal triangles and the way they are glued together. This is reduced to the question how the skein algebra behaves under gluing of boundary edges.

Suppose $a, b$ are distinct boundary edges of $\mathfrak{S}$. Let $\mathfrak{S}^{\prime}$ be the result of gluing $a$ and $b$ together in such a way that the orientation is compatible, ie $\mathfrak{S}^{\prime}=\mathfrak{S} /(a=b)$. We don't assume that $\mathfrak{S}$ is connected. It is clear that if we want to relate $\dot{\mathscr{S}}\left(\mathfrak{S}^{\prime}\right)$ to $\dot{\mathscr{S}}(\mathfrak{S})$, we have to enlarge the skein algebra to involve the boundary $\partial \mathfrak{S}$.

By a $\partial \mathfrak{S}$-tangle we mean a compact, framed, one-dimensional proper submanifold $\alpha$ of $\mathfrak{S} \times(0,1)$ such that

- at every boundary point of $\alpha$ the framing is vertical, and

- for every boundary edge $e$, the points in $\alpha \cap(e \times(0,1))$ have distinct heights, (see details in Section 2). A stated $\partial \mathfrak{S}$-tangle is a $\partial \mathfrak{S}$-tangle equipped with a map $s: \partial \alpha \rightarrow$ $\{ \pm\}$, called a state of $\alpha$.

We define the stated skein algebra $\mathscr{S}_{\mathrm{s}}(\mathfrak{S})$ to be the free $\mathcal{R}$-module spanned by the isotopy classes of stated $\partial \mathfrak{S}$-tangles modulo the usual skein relation (11), the trivial loop relation (2), and the new boundary relations (3) and (4), again see Section 2 for details.

$$
\begin{aligned}
& \boldsymbol{q}_{-}^{+}=q^{-1 / 2} \quad \uparrow, \quad \boldsymbol{q}_{+}^{+}=0, \quad \boldsymbol{q}_{-}^{-}=0 \\
& \underbrace{-}_{+}=q^{2} \underbrace{+}_{-}+q^{-1 / 2} \supset \uparrow
\end{aligned}
$$

Let $\operatorname{pr}: \mathfrak{S} \rightarrow \mathfrak{S}^{\prime}$ be the natural projection, and $c=\operatorname{pr}(a)=\operatorname{pr}(b)$. Suppose $\alpha \subset$ $\left(\mathfrak{S}^{\prime} \times(0,1)\right)$ is a stated $\partial \mathfrak{S}^{\prime}$-tangle such that

$\alpha$ is transversal to $c \times(0,1)$,

Then $\tilde{\alpha}:=\operatorname{pr}^{-1}(\alpha) \subset \mathfrak{S} \times(0,1)$ is a $\partial \mathfrak{S}$-tangle and inherits states from $\alpha$ at all boundary points, except for those in $(a \cup b) \times(0,1)$. For every $\varepsilon: \alpha \cap(c \times(0,1)) \rightarrow\{ \pm\}$ let $\tilde{\alpha}(\varepsilon)$ be the stated $\partial \mathfrak{S}$-tangle whose states on $(a \cup b) \times(0,1)$ are the lift of $\boldsymbol{\varepsilon}$.

Theorem 1. Assume $\mathfrak{S}$ is punctured bordered surface, and $\mathfrak{S}^{\prime}=\mathfrak{S} /(a=b)$, where $a, b$ are boundary edges of $\mathfrak{S}$. Let $\operatorname{pr}: \mathfrak{S} \rightarrow \mathfrak{S}^{\prime}$ be the natural projection and $c=\operatorname{pr}(a)=\operatorname{pr}(b)$.

(a) There exists a unique $\mathcal{R}$-algebra homomorphism $\rho: \mathscr{S}_{\mathbf{S}}\left(\mathfrak{S}^{\prime}\right) \rightarrow \mathscr{S}_{\mathbf{S}}(\mathfrak{S})$ such that if $\alpha$ is a $\partial \mathfrak{S}^{\prime}$-tangle satisfying (5) and (6), then

$$
\rho(\alpha)=\sum_{\varepsilon} \tilde{\alpha}(\varepsilon) .
$$


Here the sum is over all maps $\varepsilon: \alpha \cap(c \times(0,1)) \rightarrow\{ \pm\}$.

(b) In addition, $\rho$ is injective.

(c) For any 4 distinct boundary edges $a_{1}, a_{2}, b_{1}, b_{2}$ of $\mathfrak{S}$, the following diagram is commutative:

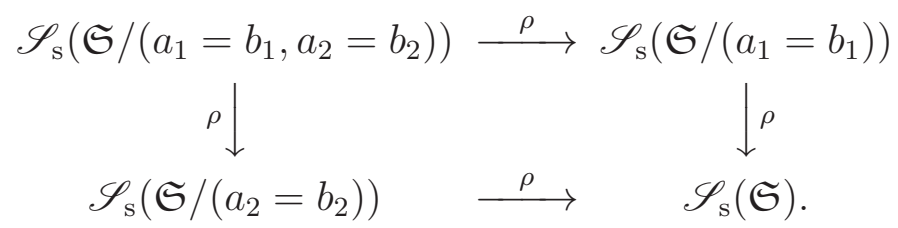

Theorem 1 is proved in Section 3 .

If we use only the skein relation (11) and the trivial loop relation (2) in the definition of $\mathscr{S}_{\mathrm{S}}(\mathfrak{S})$, then we get a bigger algebra $\widehat{\mathscr{S}_{s}}(\mathfrak{S})$, which was first introduced by Bonahon and Wong in their work [BW1] on the quantum trace map. Relation (3) was also implicitly given in [BW1, but Relation (41) is new. It is this new relation (44) which is responsible for the existence of the decomposition map $\rho: \mathscr{S}_{\mathrm{s}}\left(\mathfrak{S}^{\prime}\right) \rightarrow \mathscr{S}_{\mathrm{s}}(\mathfrak{S})$ of Theorem 1 .

We will show $\stackrel{\mathscr{S}}{(\mathfrak{S})}$ embeds naturally into $\mathscr{S}_{\mathrm{s}}(\mathfrak{S})$, which follows from the consistency of the defining relations (see Theorem 2.8 and Section 2). If we want the consistency and the well-definedness of decomposition map, then the coefficients on the right hand side of relations (3) and (4) are uniquely determined up to certain symmetries, see Section 3.4. The uniqueness makes the the definition of our skein algebra more or less canonical.

Concerning the structure of $\mathscr{S}_{\mathbf{s}}(\mathfrak{S})$, we also have the following, whose proof is given in Section 4.4.

Theorem 2. Suppose the ground ring $\mathcal{R}$ is a domain, and $\mathfrak{S}$ is a punctured bordered surface. Then $\mathscr{S}_{\mathfrak{s}}(\mathfrak{S})$ is a domain, i.e. if $x y=0$ and $x, y \in \mathscr{S}_{\mathbf{s}}(\mathfrak{S})$, then $x=0$ or $y=0$.

When $\partial \mathfrak{S}=\emptyset$, one has $\mathscr{S}_{\mathfrak{s}}(\mathfrak{S})=\dot{\mathscr{S}}(\mathfrak{S})$, the original skein algebra, and the above result had been known in this case, see [PS2, CM, [BW1, Mu].

1.3. Triangular decomposition. Suppose $\mathfrak{S}$ has an ideal triangulation $\Delta$, ie $\mathfrak{S}$ can be obtained from a finite collection $\tilde{\mathcal{F}}=\tilde{\mathcal{F}}(\Delta)$ of disjoint ideal triangles by gluing together some pairs of edges of these triangles. Choose an order of the gluing operations and apply Theorem 1 repeatedly, then we get an algebra embedding

$$
\rho_{\Delta}: \mathscr{S}_{\mathrm{S}}(\mathfrak{S}) \hookrightarrow \bigotimes_{\mathfrak{T} \in \tilde{\mathcal{F}}} \mathscr{S}_{\mathrm{S}}(\mathfrak{T})
$$

Parts (b) and (c) of Theorem 1 show that $\rho_{\Delta}$ is injective, and does not depend on the order of gluing. The map $\rho_{\Delta}$, called a triangular decomposition of $\mathscr{S}_{\mathrm{S}}(\mathfrak{S})$, can be described explicitly by a state sum formula.

It is natural now to study the stated skein algebra of an ideal triangle $\mathfrak{T}$ as every representation of $\mathscr{S}_{\mathrm{S}}(\mathfrak{T})$ gives us a representation of the stated skein algebra. In Theorem 4.6 we give an explicit presentation of the stated skein algebra of an ideal triangle, which has 12 generators with a simple set of relations. 
1.4. Application: quantum trace map. To each triangulation $\Delta$ of $\mathfrak{S}$ there is associated the Chekhov-Fock algebra $\mathcal{Y}(\Delta)$, which is built from the Chekhov-Fock algebra $\mathcal{Y}(\mathfrak{T})$ of the ideal triangle. Actually $\mathcal{Y}(\Delta)$ is a subalgebra of $\bigotimes_{\mathfrak{T} \in \tilde{\mathcal{F}}} \mathcal{Y}(\mathfrak{T})$, and is a version of the multupiplicative Chekhov-Fock algebra studied in [Liu, [BW1, Hi]. Bonahon and Wong constructed a remarkable algebra map $\operatorname{Tr}_{\Delta}: \widehat{\mathscr{S}}_{s} \rightarrow \mathcal{Y}(\Delta)$, called the quantum trace map, which when $q=1$, is the classical trace map expressing the $P S L_{2}$-trace of a curve on the surface in terms of the Thurston shear coordinates of the Teichmüller space. The existence of the quantum trace map had been conjectured in [Fo, CF2]. The construction of Bonahon and Wong is based on difficult calculations.

As an application of our triangular decomposition, we will show that the quantum trace map of Bonahon and Wong can be easily constructed using the triangular decomposition (8) as follows. First, using the explicit presentation of $\mathscr{S}_{\mathrm{S}}(\mathfrak{T})$, we construct an algebra homomorphism $\phi: \mathscr{S}_{\mathrm{s}}(\mathfrak{T}) \rightarrow \mathcal{Y}(\mathfrak{T})$. Then define $\varkappa_{\Delta}$ as the composition

$$
\varkappa_{\Delta}: \mathscr{S}_{\mathrm{S}}(\mathfrak{S}) \stackrel{\rho_{\Delta}}{\longrightarrow} \bigotimes_{\mathfrak{T} \in \tilde{\mathcal{F}}(\Delta)} \mathscr{S}_{\mathrm{S}}(\mathfrak{T}) \stackrel{\otimes \phi}{\longrightarrow} \bigotimes_{\mathfrak{T} \in \tilde{\mathcal{F}}(\Delta)} \mathcal{Y}(\mathfrak{T}) \text {. }
$$

Theorem 3. The composition $\operatorname{Tr}_{\Delta}: \widehat{\mathscr{S}_{s}}(\mathfrak{S}) \rightarrow \mathscr{S}_{\mathfrak{S}}(\mathfrak{S}) \stackrel{\varkappa_{\Delta}}{\longrightarrow} \bigotimes_{\mathfrak{T} \in \tilde{\mathcal{F}}} \mathcal{Y}(\mathfrak{T})$ coincides with the quantum trace map of Bonahon and Wong.

The proof is easy, and is given in Section 5. In essence, we replace the difficult calculations in [BW1] by explicit presentation of the stated skein algebra of the ideal triangle.

For another approach to the quantum trace map using the Muller skein algebra see [Le].

1.5. Relation to Muller's skein algebra. For a marked surface, i.e. a pair $(\overline{\mathfrak{S}}, \mathcal{P})$ where $\overline{\mathfrak{S}}$ is a compact oriented 2-dimensional manifold with (possibly empty) boundary $\partial \overline{\mathfrak{S}}$ and a finite set $\mathcal{P}$ in the boundary $\partial \overline{\mathfrak{S}}$, Muller $\left[\mathrm{Mu}\right.$ ] defines the skein algebra $\mathscr{S}^{\text {Muller }}(\overline{\mathfrak{S}}, \mathcal{P})$ using the tangles whose end points are in $\mathcal{P} \times(0,1)$. See section 6 for details. The Muller skein algebra is closed related quantum cluster algebras of marked surfaces.

Let $\mathfrak{S}=\overline{\mathfrak{S}} \backslash\left(\mathcal{P} \cup \partial^{\prime}(\overline{\mathfrak{S}})\right)$, where $\partial^{\prime}(\overline{\mathfrak{S}})$ is the union of all connected components of $\partial(\overline{\mathfrak{S}})$ not intersecting $\mathcal{P}$. Then $\mathfrak{S}$ is a punctured bordered surface. In Section 6 we show that there is a natural $\mathcal{R}$-algebra isomorphism

$$
\Omega: \mathscr{S}^{\text {Muller }}(\overline{\mathfrak{S}}, \mathcal{P}) \stackrel{\cong}{\longrightarrow} \mathscr{S}_{s,+}(\mathfrak{S}),
$$

where $\mathscr{S}_{s,+}(\mathfrak{S})$ is the subalgebra of $\mathscr{S}_{\mathrm{S}}(\mathfrak{S})$ generated by stated $\partial \mathfrak{S}$-tangles whose states are + only. Using the isomorphism $\Omega$, we can define the quantum trace map on $\mathscr{S}^{\text {Muller }}(\mathbb{S}, \mathcal{P})$

$$
\bar{\varkappa}_{\Delta}: \mathscr{S}^{\text {Muller }}(\overline{\mathfrak{S}}, \mathcal{P}) \stackrel{\Omega}{\longrightarrow} \mathscr{S}_{s,+}(\mathfrak{S}) \stackrel{\varkappa_{\Delta}}{\longrightarrow} \mathcal{Y}(\Delta)
$$

for any triangulation $\Delta$ of $\mathfrak{S}$. The following is an extension of [BW1, Proposition 29].

Theorem 4. The quantum trace map $\bar{\varkappa}_{\Delta}: \mathscr{S}^{\operatorname{Muller}}(\overline{\mathfrak{S}}, \mathcal{P}) \rightarrow \mathcal{Y}(\Delta)$ is injective.

1.6. Plan of paper. In Section 2 we give a detailed definition of the stated skein algebra $\mathscr{S}_{\mathbf{S}}(\mathfrak{S})$ of a punctured bordered surface $\mathfrak{S}$, its symmetry, filtrations, and grading. We prove Theorem 2.8 describing a natural $\mathcal{R}$-basis of $\mathscr{S}_{\mathrm{S}}(\mathfrak{S})$. The proof is a standard application of the diamond lemma. In Section 3 we prove Theorem 1, In Section 4 we give a presentation 
of $\mathscr{S}_{\mathrm{S}}(\mathfrak{S})$ when $\mathfrak{S}$ is an ideal bigon or an ideal triangle, and prove Theorem 2. We prove Theorem 3 about the quantum trace map in Section 5. In Section 6 we discuss the Muller skein algebra and prove Theorem 4 .

1.7. Acknowledgement. Much of this work is inspired by the work of Bonahon and Wong [BW1, BW2] on the quantum trace map. The author would like to thank G. Masbaum and $\mathrm{V}$. Turaev for helpful discussions. The author is partially supported by an NSF grant.

\section{Punctured Bordered surfaces And SKein Algebras}

2.1. Notations. Throughout the paper let $\mathbb{Z}$ be the set of integers, $\mathbb{N}$ the set of non-negative integers, $\mathbb{C}$ the set of complex numbers. The ground ring $\mathcal{R}$ is a commutative ring with unit 1 , containing a invertible element $q^{1 / 2}$. For a finite set $X$ we denote by $|X|$ the number of elements of $X$.

In this section we fix a punctured bordered surface $\mathfrak{S}$, i.e. a surface obtained by removing a finite set $\mathcal{P}$ from a compact oriented surface $\overline{\mathfrak{S}}$ with (possibly empty) boundary $\partial \overline{\mathfrak{S}}$, with the assumption that every connected component of the boundary $\partial \overline{\mathfrak{S}}$ has at least one point in $\mathcal{P}$. We don't require $\overline{\mathfrak{S}}$ be to connected.

Let $\partial \mathfrak{S}=\partial \overline{\mathfrak{S}} \backslash \mathcal{P}$. A connected component of $\partial \mathfrak{S}$ is called a boundary edge of $\mathfrak{S}$. Every boundary edge is diffeomorphic to the open interval $(0,1)$.

2.2. Tangles and height order. The boundary of the 3 -manifold $\mathfrak{S} \times(0,1)$ is $\partial \mathfrak{S} \times(0,1)$. For a point $(z, t) \in \mathfrak{S} \times(0,1), t$ is called its height. A vector at $(z, t)$ is called vertical if it is parallel to the $(0,1)$ factor and points in the direction of 1. A 1-dimensional submanifold $\alpha$ of $\mathfrak{S} \times(0,1)$ is framed if it is equipped with a framing, i.e. a continuous choice of a vector transverse to $\alpha$ at each point of $\alpha$.

In this paper, a $\partial \mathfrak{S}$-tangle is an unoriented, framed, compact, properly embedded 1dimensional submanifold $\alpha \subset \mathfrak{S} \times(0,1)$ such that:

- at every point of $\partial \alpha=\alpha \cap(\partial \mathfrak{S} \times(0,1))$ the framing is vertical, and

- for any boundary edge $b$, the points of $\partial_{b}(\alpha):=\partial \alpha \cap(b \times(0,1))$ have distinct heights.

Two $\partial \mathfrak{S}$-tangles are isotopic if they are isotopic in the class of $\partial \mathfrak{S}$-tangles. The emptyset, by convention, is a $\partial \mathfrak{S}$-tangle which is isotopic only to itself.

For a $\partial \mathfrak{s}$-tangle $\alpha$ define a partial order on $\partial(\alpha)$ by: $x>y$ if $x$ and $y$ are in the same boundary edge and $x$ has greater height. If $x>y$ and there is no $z$ such that $x>z>y$, we say $x$ and $y$ are consecutive.

2.3. Tangle diagrams, boundary order, positive order. As usual, $\partial \mathfrak{S}$-tangles are depicted by their diagrams on $\mathfrak{S}$, as follows. Every $\partial \mathfrak{S}$-tangle is isotopic to one with vertical framing. Suppose a vertically framed $\partial \mathfrak{S}$-tangle $\alpha$ is in general position with respect to the standard projection $\pi: \mathfrak{S} \times(0,1) \rightarrow \mathfrak{S}$, i.e. the restriction $\left.\pi\right|_{\alpha}: \alpha \rightarrow \mathfrak{S}$ is an immersion with transversal double points as the only possible singularities and there are no double points on the boundary of $\mathfrak{S}$. Then $D=\pi(\alpha)$, together with the over/underpassing information at every double point is called a $\partial \mathfrak{S}$-tangle diagram. Isotopies of (boundary ordered) $\partial \mathfrak{S}$-tangle diagrams are ambient isotopies in $\mathfrak{S}$. 
A $\partial \mathfrak{S}$-tangle diagram $D$ with a total order on each set $\partial_{b}(D):=D \cap b$, for all boundary edge $b$, is called a boundary ordered $\partial \mathfrak{S}$-tangle diagram. For example, the partial order on $\partial(\alpha)$ induces such a boundary order on $\partial D$.

Every boundary ordered $\partial \mathfrak{S}$-tangle diagram determines a unique isotopy class of the $\partial \mathfrak{S}$ tangle, where the framing is vertical everywhere. When there is no confusion, we identify a boundary ordered $\partial \mathfrak{S}$-tangle diagram with its isotopy class of $\partial \mathfrak{S}$-tangles.

Let $\mathfrak{o}$ be an orientation of $\partial \mathfrak{S}$, which on a boundary edge may or may not be equal to the orientation inherited from $\mathfrak{S}$. The $\mathfrak{o}$-order of a $\partial \mathfrak{S}$-tangle diagram $D$, is the order in which points on $\partial_{b}(D)$ are increasing when going along the direction of $\mathfrak{o}$. It is clear that every $\partial \mathfrak{S}$-tangle, after an isotopy, can be presented by an $\mathfrak{o}$-ordered $\partial \mathfrak{S}$-tangle diagram, i.e. a $\partial \mathfrak{S}$-tangle diagram with $\mathfrak{o}$-order.

If $\mathfrak{o}$ is the orientation coming from $\mathfrak{S}$, the $\mathfrak{o}$-order is called the positive order. Every isotopy class of $\partial \mathfrak{S}$-tangles can be presented by a positively ordered $\partial \mathfrak{S}$-tangle diagram.

2.4. Framed Reidemeister moves. Every isotopy class of $\partial \mathfrak{S}$-tangle can be presented by infinitely many boundary ordered $\partial \mathfrak{S}$-tangle diagrams. Just like in the theory of framed links, two positively ordered $\partial \mathfrak{S}$-tangle diagrams represent isotopic $\partial \mathfrak{S}$-tangles if and only if one can be obtained from the other by a sequence of moves, each is either an isotopy in $\mathfrak{S}$ or one of the framed Reidemeister moves RI, RII, and RIII, described in Figure 1.

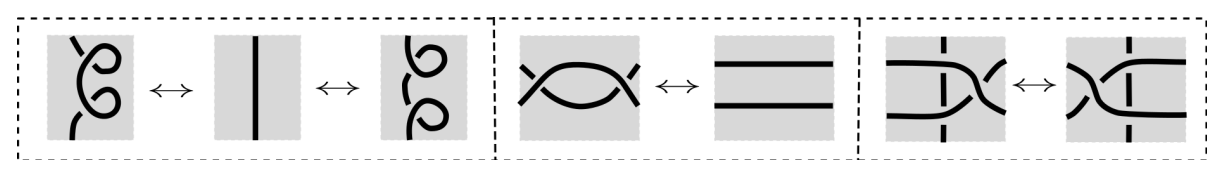

Figure 1. Framed Reidemeister moves RI, RII, and RIII

If we don't restrict to positive order, then two boundary ordered $\partial \mathfrak{S}$-tangle diagrams represent isotopic $\partial \mathfrak{S}$-tangles if and only if one can be obtained from the other by a sequence of moves, each is either an isotopy in $\mathfrak{S}$, one of RI, RII, RIII, and the exchange move described in Figure 2.

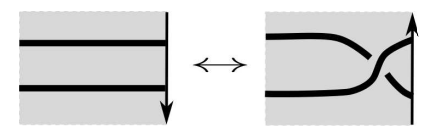

Figure 2. Exchange move. Here the arrowed interval is a part of a boundary edge, and the order on that part is such that the point closer to the tip of the arrow is higher. Besides, these two points are consecutive in the height order.

2.5. Stated skein module/algebra. A stated $\partial \mathfrak{S}$-tangle $\alpha$ is a $\partial \mathfrak{S}$-tangle $\alpha$ equipped with a state, which is a function $s: \partial \alpha \rightarrow\{+,-\}$. Similarly, a stated $\partial \mathfrak{S}$-tangle diagram $D$ is an $\partial \mathfrak{S}$-tangle diagram $D$ equipped with a state $s: \partial D \rightarrow\{ \pm\}$. 
The (Kauffman bracket) stated skein module $\mathscr{S}_{\mathbf{s}}(\mathfrak{S})$ is the $\mathcal{R}$-module freely spanned by isotopy classes of stated $\partial \mathfrak{S}$-tangles modulo the defining relations, which are the skein relation (10), the trivial loop relation (11), and the boundary relations (12) and (13):

$$
\begin{aligned}
& Y=q)\left(+q^{-1} \Longleftarrow\right. \\
& \mathrm{O}=\left(-q^{2}-q^{-2}\right) \\
& \boldsymbol{q}_{-}^{+}=q^{-1 / 2} \quad \uparrow, \quad \mathfrak{q}_{+}^{+}=0, \quad \mathfrak{q}_{-}^{-}=0 \\
& \beth_{+}^{-}=q^{2} \underbrace{+}_{-}+q^{-1 / 2} \supset \uparrow
\end{aligned}
$$

Here is the convention about pictures in these identities, as well as in other identities in this paper. Each shaded part is a part of $\mathfrak{S}$, with a stated $\partial \mathfrak{S}$-tangle diagram on it. Each arrowed line is part of a boundary edge, and the order on that part is indicated by the arrow and the points on that part are consecutive in the height order. The order of other end points away from the picture can be arbitrary and are not determined by the arrows of the picture.

Relation (10) says that if 3 boundary ordered, stated $\partial \mathfrak{S}$-tangle diagrams $D_{1}, D_{2}, D_{3}$ are identical everywhere, except for a small disk in which $D_{1}, D_{2}, D_{3}$ are like in respectively the first, the second, and the third shaded areas, then $\left[D_{1}\right]=q\left[D_{2}\right]+q^{-1}\left[D_{3}\right]$ in the skein module $\mathscr{S}_{\mathbf{S}}(\mathfrak{S})$. Here $\left[D_{i}\right]$ is the isotopy class of the stated $\partial \mathfrak{S}$-tangle determined by $D_{i}$. Other relations are interpreted similarly.

For two $\partial \mathfrak{S}$-tangles $\alpha_{1}$ and $\alpha_{2}$ the product $\alpha_{1} \alpha_{2}$ is defined as the result of stacking $\alpha_{1}$ above $\alpha_{2}$. That is, first isotope $\alpha_{1}$ and $\alpha_{2}$ so that $\alpha_{1} \subset \mathfrak{S} \times(1 / 2,1)$ and $\alpha_{2} \subset \mathfrak{S} \times(0,1 / 2)$. Then $\alpha_{1} \alpha_{2}=\alpha_{1} \cup \alpha_{2}$. It is easy to see that this gives rise to a well defined product and hence an $\mathcal{R}$-algebra structure on $\mathscr{S}_{\mathbf{s}}(\mathfrak{S})$.

It is clear that if $\mathfrak{S}_{1}$ and $\mathfrak{S}_{2}$ are two punctured bordered surfaces, then there is a natural isomorphism

$$
\mathscr{S}_{\mathrm{s}}\left(\mathfrak{S}_{1} \sqcup \mathfrak{S}_{2}\right) \cong \mathscr{S}_{\mathrm{s}}\left(\mathfrak{S}_{1}\right) \otimes_{\mathcal{R}} \mathscr{S}_{\mathrm{s}}\left(\mathfrak{S}_{2}\right)
$$

Remark 2.1. If we don't impose the boundary relations (12) and (13), then we get a bigger skein module $\widehat{\mathscr{S}_{s}}(\mathfrak{S})$, which was first introduced in [BW1]. Of course $\mathscr{S}_{\mathrm{S}}(\mathfrak{S})$ is a quotient of $\widehat{\mathscr{S}_{s}}(\mathfrak{S})$. If $\partial \mathfrak{S}=\emptyset$, then $\mathscr{S}_{\mathrm{S}}(\mathfrak{S})=\widehat{\mathscr{S}}_{s}(\mathfrak{S})=\dot{\mathscr{S}}(\mathfrak{S})$.

Remark 2.2. If $\mathfrak{S}$ is allowed to have a closed boundary component, then unless $q^{2}=1$, the defining relations are not consistent and the skein module $\mathscr{S}_{\mathrm{s}}(\mathfrak{S})$ is small.

2.6. Consequences of defining relations. Define $C_{\varepsilon^{\prime}}^{\varepsilon}$ for $\varepsilon, \varepsilon^{\prime} \in\{ \pm\}$ by

$$
C_{+}^{+}=C_{-}^{-}=0, \quad C_{-}^{+}=q^{-1 / 2}, \quad C_{+}^{-}=-q^{-5 / 2} .
$$


Lemma 2.3. In $\mathscr{S}_{\mathrm{s}}(\mathfrak{S})$ one has

$$
\begin{aligned}
-q^{-3} \boldsymbol{\rho} & =\mid=-q^{3} \mathbf{Q}^{\mathbf{\prime}} \\
\mathcal{G}_{\varepsilon^{\prime}}^{\varepsilon} & =C_{\varepsilon^{\prime}}^{\varepsilon} \uparrow \\
\varepsilon_{\varepsilon}^{\prime} \rho & =\nabla_{\varepsilon^{\prime}}^{\varepsilon}=-q^{3} C_{\varepsilon}^{\varepsilon^{\prime}} \uparrow
\end{aligned}
$$

Proof. Identity (16) follows from the skein relation and the trivial loop relation, see [Kau].

Except for $\left(\varepsilon, \varepsilon^{\prime}\right)=(-,+)$, (17) is a defining relation. Applying (13), then (11), then (12),

$$
\mathcal{q}_{+}^{-}=q^{2} \mathcal{q}_{-}^{+}+q^{-1 / 2} \bigcirc \uparrow=q^{2}\left(q^{-1 / 2}\right)+q^{-1 / 2}\left(-q^{2}-q^{-2}\right)=-q^{-5 / 2}
$$

which proves the remaining case of (17).

The first equality of (18) follows from a rotation by $\pi$. Using isotopy, we have

$$
\mathcal{G}_{\varepsilon^{\prime}}^{\varepsilon}=\mathcal{C}_{\varepsilon}^{\varepsilon^{\prime}}=-q^{3} \mathcal{G}_{\varepsilon^{\prime}}^{\varepsilon}=-q^{3} C_{\varepsilon^{\prime}}^{\varepsilon}
$$

where the 2nd and the 3rd identities follow from (16) and (17).

Lemma 2.4 (Height exchange move). (a) One has

$$
\begin{aligned}
& \beth_{+}^{+}=q^{-1}\left(\beth_{+}^{+}\right), \quad \square_{-}^{-}=q^{-1}\left(\varpi_{-}^{-}\right), \quad \square_{-}^{+}=q\left(\beth_{-}^{+}\right) \\
& q^{\frac{3}{2}} \beth_{+}^{-}-q^{-\frac{3}{2}} \beth_{+}^{-}=\left(q^{2}-q^{-2}\right) \supset \uparrow .
\end{aligned}
$$

(b) Consequently, if $q=1$ or $q=-1$, then for all $\varepsilon, \varepsilon^{\prime} \in\{ \pm\}$,

$$
\beth_{\varepsilon^{\prime}}^{\varepsilon}=q \overbrace{}^{\varepsilon} \varepsilon^{\prime} \text {. }
$$

Proof. (a) Using isotopy, then skein relation (10), and then (17), we have

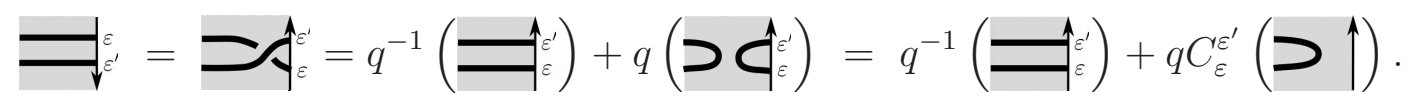

When $\varepsilon=\varepsilon^{\prime}, C_{\varepsilon}^{\varepsilon^{\prime}}=0$, and (22) proves the first two identities of (19).

Suppose $\varepsilon=+, \varepsilon^{\prime}=-$. Using (22), then (13) and (11), we have

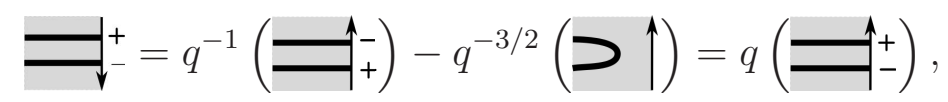

proving the last identity of (19). Now suppose $\varepsilon=+, \varepsilon^{\prime}=-$. Rewrite (13) in the form

$$
\vec{\beth}_{-}^{+}=q^{-2} \underline{-}_{+}^{-}-q^{-5 / 2} \supset \uparrow
$$

Using (23) in (22), we get (20).

(b) follows from (a).

Corollary 2.5. If $q=1$, then $\mathscr{S}_{\mathbf{s}}(\mathfrak{S})$ is commutative.

Proof. When $q=1$, Identity (21) shows that the height order does not matter in $\mathscr{S}_{\mathrm{s}}(\mathfrak{S})$. Besides, the skein relation show that over-crossing is the same as under-crossing. Hence for any two $\partial \mathfrak{S}$-tangles $\alpha, \beta$, we have $\alpha \beta=\beta \alpha$. 
Remark 2.6. In general, because of relation (21), $\mathscr{S}_{\mathfrak{S}}(\mathfrak{S})$ is not commutative when $q=-1$. For example, when $\mathfrak{S}$ is an ideal triangle, $\mathscr{S}_{\mathfrak{s}}(\mathfrak{S})$ is not commutative when $q=-1$. This should be contrasted with the case of the usual skein algebra $\mathscr{\mathscr { S }}(\mathfrak{S})$, which is commutative and is canonically equal to the $S L_{2}(\mathbb{C})$ character variety of $\pi_{1}(\mathfrak{S})$ if $\mathcal{R}=\mathbb{C}$ and $q=-1$ (assuming $\mathfrak{S}$ is connected), see [Bul, [PS1].

\subsection{Reflection anti-involution.}

Proposition 2.7. Suppose $\mathcal{R}=\mathbb{Z}\left[q^{ \pm 1 / 2}\right]$. There exists a unique $\mathbb{Z}$-linear anti-automorphism $\chi: \mathscr{S}_{\mathfrak{S}}(\mathfrak{S}) \rightarrow \mathscr{S}_{\mathrm{S}}(\mathfrak{S})$, such that $\chi\left(q^{1 / 2}\right)=q^{-1 / 2}$ and $\chi(\alpha)=\bar{\alpha}$, where $\alpha$ is a stated $\partial \mathfrak{S}$-tangle, and $\bar{\alpha}$ the image of $\alpha$ under the reflection of $\mathfrak{S} \times(0,1)$, defined by $(z, t) \rightarrow(z, 1-t)$. Here $\chi$ is an anti-automorphism means for any $x, y \in \mathscr{S}_{\mathbf{s}}(\mathfrak{S})$ and $r \in \mathcal{R}$,

$$
\chi(x+y)=\chi(x)+\chi(y), \quad \chi(x y)=\chi(y) \chi(x) .
$$

Proof. Since $\mathscr{S}_{\mathrm{S}}(\mathfrak{S})$ is spanned by stated $\partial \mathfrak{S}$-tangles, the uniqueness is clear.

Let $\mathcal{L}$ be the free $\mathcal{R}$-module with basis the set of isotopy classes of stated $\partial \mathfrak{S}$-tangles and $\tilde{\chi}: \mathcal{L} \rightarrow \mathcal{L}$ be the $\mathbb{Z}$-linear map defined by $\tilde{\chi}(r \alpha)=\bar{r} \bar{\alpha}$, where for $r \in \mathcal{R}, \bar{r}$ is the image of $r$ under the involution $q^{1 / 2} \rightarrow q^{-1 / 2}$. Using the height exchange move (Lemma 2.4), one sees that $\chi$ respects all the defining relations, and hence descends to a map $\chi: \mathscr{S}_{\mathbb{S}}(\mathfrak{S}) \rightarrow \mathscr{S}_{\mathrm{s}}(\mathfrak{S})$. It is clear that $\chi$ is an anti-automorphism.

Clearly $\chi^{2}=\mathrm{id}$. We call $\chi$ the reflection anti-involution.

2.8. Basis of stated skein module. A $\partial \mathfrak{S}$-tangle diagram $D$ is simple if it has neither double point nor trivial component. Here a closed component of $D$ is trivial if it bounds a disk in $\mathfrak{S}$, and an arc component of $\alpha$ is trivial if it can be homotoped relative its boundary, in the complement of other components of $D$, to a subset of a boundary edge. By convention, the empty set is considered as a simple stated $\partial \mathfrak{S}$-tangle diagram with 0 component.

We order the set $\{ \pm\}$ so that + is greater than - A state $s: \partial D \rightarrow\{ \pm\}$ of a boundary ordered $\partial \mathfrak{S}$-tangle diagram $D$ is increasing if for any $x, y \in \partial D$ with $x \geq y$, one has $s(x) \geq s(y)$. Thus, in an increasing state, on any boundary edge, the points with + state are above all the points with - state.

Let $B(\mathfrak{S})$ be the set of of all isotopy classes of increasingly stated, positively ordered, simple $\partial \mathfrak{S}$-tangle diagrams.

Theorem 2.8. As an $\mathcal{R}$-module, $\mathscr{S}_{\mathbf{s}}(\mathfrak{S})$ is free with basis $B(\mathfrak{S})$.

Proof. The proof uses the diamond lemma, in the form explained in [SW]. For a set $X$ denote by $\mathcal{R} X$ the free $\mathcal{R}$-module with basis $X$, with the convention $\mathcal{R} X=\{0\}$ when $X=\emptyset$. In this proof, all the $\partial \mathfrak{S}$-tangle diagram is assumed to have positive order.

Let $\tilde{B}$ be the set of all isotopy classes of stated $\partial \mathfrak{S}$-tangle diagrams. Then $B=B(\mathfrak{S})$ is a subset of $\tilde{B}$. Define a binary relation $\rightarrow$ on $\mathcal{R} \tilde{B}$ as follows. First assume $D \in V$ and $E \in \mathcal{R} \tilde{B}$. We write $D \rightarrow E$ if $D$ is any element in $\tilde{B}$ presented by the left hand side of an identity in the defining relations (10)-(13), and $E$ is the corresponding right hand side.

Now assume $E^{\prime}, E^{\prime \prime} \in \mathcal{R} \tilde{B}$, with $E^{\prime}=\sum_{i=1}^{k} c_{i} D_{i}$, where $D_{i} \in \tilde{B}$. We write $E^{\prime} \rightarrow E^{\prime \prime}$ if there is an index $j \leq k$ and $E \in \mathcal{R} \tilde{B}$ with $D_{j} \rightarrow E$, such that $E^{\prime \prime}$ is obtained from $E^{\prime}$ by replacing $D_{j}$ with $E$ in the sum $\sum_{i=1}^{k} c_{i} D_{i}$. 
Let $\stackrel{\star}{\longrightarrow}$ be the reflexive and transitive relation on $\mathcal{R} \tilde{B}$ generated by $\rightarrow$, i.e. $E \stackrel{\star}{\longrightarrow} E^{\prime}$ if either $E=E^{\prime}$ or there are $E_{1}, E_{2}, \ldots, E_{k} \in \mathcal{R} \tilde{B}$ with $E_{i} \rightarrow E_{i+1}$ for all $i=1, \ldots, k-1$ such that $E_{1}=E, E_{k}=E^{\prime}$. If $E \stackrel{\star}{\longrightarrow} E^{\prime}$, we say $E^{\prime}$ is a descendant of $E$.

Let $\sim$ be the equivalence relation on $\mathcal{R} V$ generated by $\rightarrow$. We will prove the following two lemmas in Subsection 2.9.

Lemma 2.9. One has $\mathcal{R} \tilde{B} / \sim=\mathscr{S}_{\mathrm{s}}(\mathfrak{S})$.

Lemma 2.10. The relation $\rightarrow$ is

(i) terminal, i.e. there does not exist an infinite sequence $E_{1} \rightarrow E_{2} \rightarrow E_{3} \rightarrow \ldots$, and

(ii) locally confluent on $\tilde{B}$, i.e. if $D \rightarrow E_{1}$ and $D \rightarrow E_{2}$ for some $D \in \tilde{B}$, then $E_{1}, E_{2}$ have a common descendent.

Since $\rightarrow$ is terminal and locally confluent on $\tilde{B}$, [SW. Theorem 2.3] shows that $\tilde{B}_{i r r}$, the subset of elements $D \in \tilde{B}$ for which there is no $E \in \mathcal{R} \tilde{B}$ such that $D \rightarrow E$, is a basis of $\mathcal{R} \tilde{B} / \sim$, which is $\mathscr{S}_{\mathrm{s}}(\mathfrak{S})$ (by Lemma 2.9). It remains to notice that $\tilde{B}_{i r r}=B$. The theorem is proved.

\subsection{Proofs of Lemmas 2.9 and 2.10.}

Proof of Lemma 2.9. Two stated $\partial \mathfrak{S}$-tangle diagrams define the same stated $\partial \mathfrak{S}$-tangle if and only if one can be obtained from the other by a sequence of framed Reidemeister moves RI, RII and RIII moves of Figure 1. Thus, $\mathscr{S}_{\mathrm{s}}(\mathfrak{S})=\mathcal{R} \tilde{B} /(\mathrm{rel})$, where $(\mathrm{rel})$ consists of the defining relations (10)-(13) and the the moves RI, RII, RIII. But the three moves RI, RII, and RIII are consequences of the skein relation (10) and the trivial knot relation (11) (see $[\mathrm{Kau}]$ ). Hence, $\mathscr{S}_{\mathrm{s}}(\mathfrak{S})=\mathcal{R} \tilde{B} /($ rel $)=\mathcal{R} \tilde{B} / \sim$.

Suppose $s: \partial(\alpha) \rightarrow\{ \pm\}$ is a state of a $\partial \mathfrak{S}$-tangle $\alpha$. A pair $(x, y) \in \partial(\alpha)^{2}$ is $s$-decreasing if $x>y$ and $s(x)=-, s(y)=+$. Let nd $(s)$ be the number of $s$-decreasing pairs. Then $\operatorname{nd}(s)=0$ if and only if $s$ is increasing.

Proof of Lemma 2.10. (a) For $D \in \tilde{B}$, with state $s$, let $\operatorname{deg}(D)$ be the sum of 4 terms: two times the number of double points, the number of components, the number of boundary points, and $\operatorname{nd}(D)$. By checking each of the relations (10)-(13) , one sees that that if $D \in \tilde{B}$ and $D \rightarrow E \in \mathcal{R} \tilde{B}$, then $E$ is a linear combination of elements $D_{j} \in \tilde{B}$ with $\operatorname{deg}\left(D_{j}\right)<$ $\operatorname{deg}(D)$. Hence, By [SW, Theorem 2.2] the relation $\rightarrow$ is terminal.

(b) Suppose $D$ is a stated $\partial \mathfrak{S}$-tangle diagram. For now we don't consider $D$ up to isotopy. A disk $d \subset \mathfrak{S}$ is called D-applicable if $D \cap d$ is the left hand side of one of the defining relations (10)-(13). In that case let $F_{d}(D)=\sum_{j} c_{j} D_{j}$ be the corresponding right hand side, so that $D \rightarrow F_{d}(D)$. Here $D_{j}=D$ outside $d$.

Suppose $E=\sum c_{i} D_{i}$, where $0 \neq c_{i} \in \mathcal{R}$ and $D_{i}$ is a stated $\partial \mathfrak{S}$-tangle diagram for each $i$. A disk $d \subset \mathfrak{S}$ is said to be $E$-applicable if $d$ is $D_{i}$-applicable for each $i$. In that case, define $F_{d}(E)=\sum c_{i} F_{d}\left(D_{i}\right)$. Clearly $E \stackrel{\star}{\longrightarrow} F_{d}(E)$.

If $d_{1}, d_{2}$ are two disjoint $D$-applicable disks, then $d_{1}$ is $F_{d_{2}}(D)$-applicable and $d_{2}$ is $F_{d_{1}}(D)$ applicable, and $F_{d_{1}}\left(F_{d_{2}}(D)\right)=F_{d_{2}}\left(F_{d_{1}}(D)\right)$ is a common descendant of $F_{d_{1}}(D)$ and $D_{d_{2}}(D)$.

Now suppose $D \rightarrow E_{1}$ and $D \rightarrow E_{2}$. We have to show that $E_{1}$ and $E_{2}$ have a common descendant. There are applicable $D$-disks $d_{1}$ and $d_{2}$ such that $E_{1}=F_{d_{1}}(D)$ and $E_{2}=F_{d_{2}}(D)$. 
If $d_{1}$ and $d_{2}$ are disjoint, then $E_{1}$ and $E_{2}$ have a common descendant. It remains the case when $d_{1} \cap d_{2} \neq \emptyset$.

The support of a $D$-applicable disk $d$ is defined to be

- the double point for the case of (10),

- the closed disk bounded by the loop for the case of (11)

- the closed disk bounded the arc of $D$ and part of the boundary edge between the two end points of the arc in the case of (12), and

- the closed interval between the two boundary points on the boundary edge in the case of (13) .

In doing the move $D \rightarrow F_{d}(D)$, we can assume that $d$ is a small neighborhood of its support. Hence if the supports of $d_{1}$ and $d_{2}$ are disjoint, then we can assume that $d_{1}$ and $d_{2}$ are disjoint. By inspecting the left hand sides of (10)-(13) we see that there are only three cases when supports of $d_{1}$ and $d_{2}$ are not disjoint. These cases are described in Figures 3 5.

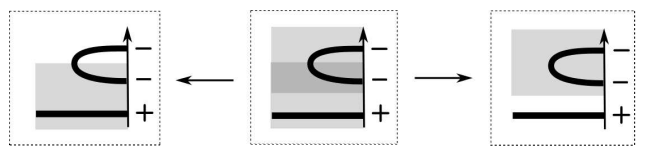

Figure 3. Case 1: The shaded area on the right (resp. left) is $d_{1}$ (resp. $\left.d_{2}\right)$. The shaded area in the middle is $d_{1} \cup d_{2}$

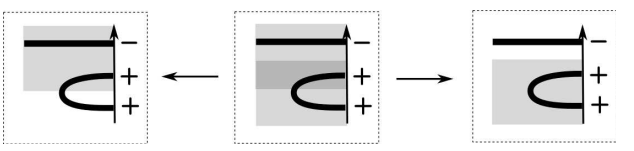

Figure 4. Case 2: The shaded area on the right (resp. left) is $d_{1}$ (resp. $d_{2}$ ).

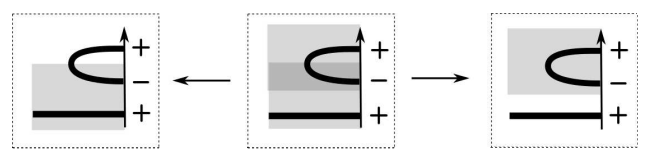

Figure 5. Case 3: The shaded area on the right (resp. left) is $d_{1}$ (resp. $\left.d_{2}\right)$.

Note that the proof of (17) actually shows that

$$
\mathcal{\bigotimes}_{+}^{-} \stackrel{\star}{\longrightarrow}-q^{-5 / 2} \uparrow
$$

Case 1. From (12) we have $E_{1}=F_{d_{1}}(D)=0$. Using (13) then (24),

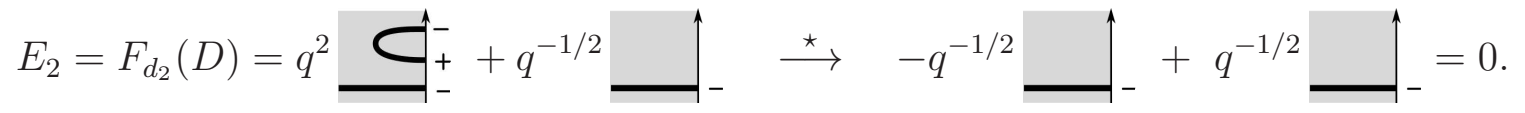

Case 2. From (12) we have $E_{1}=F_{d_{1}}(D)=0$. Using (13) then (24),

$$
E_{2}=F_{d_{2}}(D)=q^{2} \vec{\square}_{+}^{+}+q^{-1 / 2} \longrightarrow+\left.\right|^{+} \quad-q^{-1 / 2} \longrightarrow^{+}+\left.q^{-1 / 2}\right|^{+}=0 .
$$


Case 3. From (12) we have

$$
E_{1}=F_{d_{1}}(D)=q^{-1 / 2} \uparrow_{+}
$$

Using (12), we have

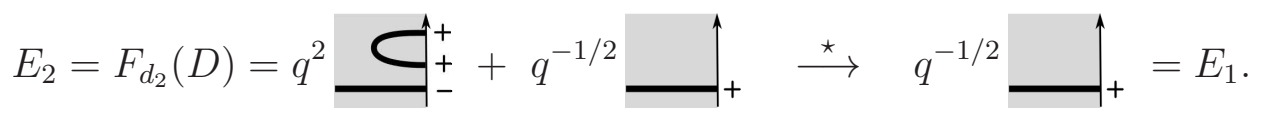

In all three cases, $E_{1}$ is a common descendant of $E_{1}$ and $E_{2}$, completing the proof.

2.10. More general boundary order. Let $\mathfrak{o}$ be an orientation of $\partial \mathfrak{S}$. For a boundary edge $b$, we say $\mathfrak{o}$ is positive on $b$ if it is equal to the orientation inherited from $\mathfrak{S}$, otherwise it is called negative on $b$. Equation (13) can be rewritten as (23), which expresses a positive order term as a sum of a negative order term and a term of lesser complexity. Let $B(\mathfrak{o} ; \mathfrak{S})$ be the set of of all isotopy classes of increasingly stated, $\mathfrak{o}$-ordered, simple $\partial \mathfrak{S}$-tangle diagrams. The proof of Theorem 2.8 can be easily modified to give the following more general statement.

Theorem 2.11. Suppose $\mathfrak{S}$ is a punctured bordered surface and $\mathfrak{o}$ is an orientation of $\partial \mathfrak{S}$. Then $B(\mathfrak{o} ; \mathfrak{S})$ is an $\mathcal{R}$-basis of $\mathscr{S}_{\mathbf{S}}(\mathfrak{S})$.

2.11. Filtration. Note that $|\partial(\alpha)|$ is even, for any $\partial \mathfrak{S}$-tangle $\alpha$. For each non-negative integer $m$ let $F_{m}=F_{m}\left(\mathscr{S}_{\mathrm{s}}(\mathfrak{S})\right)$ be the $\mathcal{R}$-submodule of $\mathscr{S}_{\mathrm{s}}(\mathfrak{S})$ spanned by all $\partial \mathfrak{S}$-tangles $\alpha$ such that $|\partial(\alpha)| \leq 2 m$. Clearly $F_{m} \subset F_{m+1}$ and $F_{m} F_{k} \subset F_{m+k}$. In other words, $\mathscr{S}_{\mathrm{s}}(\mathfrak{S})$ is a filtered algebra with the filtration $\left\{F_{m}\right\}$. The associated graded algebra is denoted by $\operatorname{Gr}\left(\mathscr{S}_{\mathrm{s}}(\mathfrak{S})\right)$, with $\operatorname{Gr}_{m}\left(\mathscr{S}_{\mathrm{s}}(\mathfrak{S})\right)=F_{m} / F_{m-1}$ for $m \geq 1$ and $\operatorname{Gr}_{0}=F_{0}$. The following is a consequence of Theorem 2.11.

Proposition 2.12. Le $\mathfrak{S}$ be a punctured bordered surface and $\mathfrak{o}$ be an orientation of $\partial \mathfrak{S}$.

(a) The set $\{\alpha \in B(\mathfrak{o} ; \mathfrak{S})|| \partial(\alpha) \mid \leq 2 m\}$ is an $\mathcal{R}$-basis of $F_{m}\left(\mathscr{S}_{\mathfrak{s}}(\mathfrak{S})\right.$ ).

(b) The set $\{\alpha \in B(\mathfrak{o} ; \mathfrak{S})|| \partial(\alpha) \mid=2 m\}$ is an $\mathcal{R}$-basis of $\operatorname{Gr}_{m}\left(\mathscr{S}_{\mathbf{s}}(\mathfrak{S})\right)$.

2.12. Grading. For a boundary edge $b$ and a stated $\partial \mathfrak{S}$-tangle $\alpha$ define

$$
\boldsymbol{\delta}_{\alpha}(b)=\sum_{u \in \partial_{b}(\alpha)} s(u) \in \mathbb{Z},
$$

where, as usual, we identify + with +1 and - with -1 . Let $\mathcal{E}_{\partial}$ be the set of all boundary edges. Then $\boldsymbol{\delta}_{\alpha} \in \mathbb{Z}^{\mathcal{E}_{\partial}}$, the set of all maps $\mathcal{E}_{\partial} \rightarrow \mathbb{Z}$.

For $\mathbf{k} \in \mathbb{Z}^{\mathcal{E}_{\partial}}$ let $G_{\mathbf{k}}=G_{\mathbf{k}}\left(\mathscr{S}_{\mathfrak{s}}(\mathfrak{S})\right)$ be the $\mathcal{R}$-submodule of $\mathscr{S}_{\mathfrak{s}}(\mathfrak{S})$ spanned by all stated $\partial \mathfrak{S}$ tangles $\alpha$ such that $\boldsymbol{\delta}(\alpha)=\mathbf{k}$. From the defining relations it is clear that $\mathscr{S}_{\mathbf{s}}(\mathfrak{S})=\bigoplus_{\mathbf{k} \in \mathbb{Z}^{l}} G_{\mathbf{k}}$ and $G_{\mathbf{k}} G_{\mathbf{k}^{\prime}} \subset G_{\mathbf{k}+\mathbf{k}^{\prime}}$. In other words, $\mathscr{S}_{\mathfrak{s}}(\mathfrak{S})$ is a graded algebra with the grading $\left\{G_{\mathbf{k}}, k\right\}$.

Fix a boundary edge $b$. For $k \in \mathbb{Z}$ let $G_{b, k}=G_{b, k}\left(\mathscr{S}_{\mathbf{s}}(\mathfrak{S})\right)$ be the $\mathcal{R}$-submodule of $\mathscr{S}_{\mathrm{s}}(\mathfrak{S})$ spanned by all stated $\partial \mathfrak{S}$-tangles $\alpha$ such that $\boldsymbol{\delta}_{\alpha}(b)=k$. Again $\mathscr{S}_{\mathfrak{s}}(\mathfrak{S})=\bigoplus \in \mathbb{Z} G_{b, k}$ and $G_{b, k} G_{b, k^{\prime}} \subset G_{b, k+k^{\prime}}$. In other words, $\mathscr{S}_{\mathbf{s}}(\mathfrak{S})$ is a $\mathbb{Z}$-graded algebra with the grading $\left\{G_{b, k}\right\}$. The following is a consequence of Theorem 2.11.

Proposition 2.13. Le $\mathfrak{S}$ be a punctured bordered surface and $\mathfrak{o}$ be an orientation of $\partial \mathfrak{S}$.

(a) The set $\left\{\alpha \in B(\mathfrak{o} ; \mathfrak{S}) \mid \boldsymbol{\delta}_{\alpha}=\mathbf{k}\right\}$ is an $\mathcal{R}$-basis of $G_{\mathbf{k}}\left(\mathscr{S}_{\mathbf{s}}(\mathfrak{S})\right)$.

(b) The set $\left\{\alpha \in B(\mathfrak{o} ; \mathfrak{S}) \mid \boldsymbol{\delta}_{\alpha}(b)=k\right\}$ is an $\mathcal{R}$-basis of $G_{b, k}\left(\mathscr{S}_{\mathbf{S}}(\mathfrak{S})\right)$. 
The $\mathbb{Z}$-grading $\left\{G_{b, k}\right\}$ allows to define the b-leading term $\operatorname{lt}_{b}(x)$ of non-zero $x \in \mathscr{S}_{\mathbb{s}}(\mathfrak{S})$. Suppose $x=\sum_{j} c_{j} D_{j}$, where $0 \neq c_{j} \in \mathcal{R}$ and $D_{j} \in B(\mathfrak{o} ; \mathfrak{S})$. Assume $k=\max _{j} \boldsymbol{\delta}_{D_{j}}(b)$. Define

$$
\mathrm{lt}_{b}(x)=\sum_{\delta_{D_{j}}(b)=k} c_{j} D_{j}
$$

2.13. The ordinary skein algebra $\mathscr{\mathscr { S }}(\mathfrak{S})$. Recall that the ordinary skein algebra $\mathscr{\mathscr { S }}(\mathfrak{S})$ is the $\mathcal{R}$-module freely spanned by isotopy classes of framed links in $\mathfrak{S} \times(0,1)$ modulo the skein relation (10) and the trivial loop relation (11). The $\operatorname{map~id}_{*}: \mathscr{S}(\mathfrak{S}) \rightarrow \mathscr{S}_{\mathrm{s}}(\mathfrak{S})$, defined on a framed link $\alpha$ by $\operatorname{id}_{*}(\alpha)=\alpha$, is an $\mathcal{R}$-algebra homomorphism.

Corollary 2.14. The map $\operatorname{id}_{*}: \mathscr{S}(\mathfrak{S}) \rightarrow \mathscr{S}_{\mathrm{s}}(\mathfrak{S})$ is an embedding, and $\operatorname{id}_{*}(\dot{\mathscr{S}}(\mathfrak{S}))=$ $F_{0}\left(\mathscr{S}_{\mathrm{s}}(\mathfrak{S})\right)$.

Proof. The manifold $\mathfrak{S}:=\mathfrak{S} \backslash \partial \mathfrak{S}$ is also a punctured bordered surface without boundary. It is clear that $\mathscr{\mathscr { S }}(\mathfrak{S})=\mathscr{S}_{\mathrm{s}}(\mathfrak{S})$, and the $\mathcal{R}$-basis of the latter described by Theorem 2.8 is the $\mathcal{R}$-basis of $F_{0}\left(\mathscr{S}_{\mathrm{s}}(\mathfrak{S})\right)$. The result follows.

\section{Decomposing and gluing Punctured bordered surfaces}

3.1. Gluing punctured bordered surfaces. Suppose $a$ and $b$ are distinct boundary edges of a punctured bordered surface $\mathfrak{S}$. Let $\mathfrak{S}^{\prime}=\mathfrak{S} /(a=b)$, the result of gluing $a$ and $b$ together in such a way that the orientation is compatible. The canonical projection pr: $\mathfrak{S} \rightarrow \mathfrak{S}^{\prime}$ induces a projection $\widetilde{\operatorname{pr}}: \mathfrak{S} \times(0,1) \rightarrow \mathfrak{S}^{\prime} \times(0,1)$. Let $c=\operatorname{pr}(a)=\operatorname{pr}(b)$.

A $\partial \mathfrak{S}^{\prime}$-tangle $\alpha \subset\left(\mathfrak{S}^{\prime} \times(0,1)\right)$, is said to be vertically transverse to $c$ if

- $\alpha$ is transversal to $c \times(0,1)$,

- the points in $\partial_{c} \alpha:=\alpha \cap(c \times(0,1))$ have distinct heights, and have vertical framing.

Suppose $\alpha$ is a $\partial \mathfrak{S}^{\prime}$-tangle vertically transversal to $c$. Then $\tilde{\alpha}:=\widetilde{\operatorname{pr}}^{-1}(\alpha)$ is a $\partial \mathfrak{S}$-tangle. Suppose in addition $\alpha$ is stated, with state $s: \partial \alpha \rightarrow\{ \pm\}$. For any $\varepsilon: \alpha \cap(c \times(0,1)) \rightarrow\{ \pm\}$ define $\tilde{\alpha}(\varepsilon)$ be $\tilde{\alpha}$ equipped with state $\tilde{s}$ defined by $\tilde{s}(x)=s(\operatorname{pr}(x))$ if $\operatorname{pr}(x) \in \partial \alpha$ and $\tilde{s}(x)=\varepsilon(\operatorname{pr}(x))$ if $\operatorname{pr}(x) \in c$. We call $\tilde{\alpha}(\varepsilon)$ a lift of $\alpha$. If $|\alpha \cap(c \times(0,1))|=k$, then $\alpha$ has $2^{k}$ lifts.

3.2. Proof of Theorem 1, For the reader convenience we reformulate Theorem 1 here.

Theorem 3.1. Suppose $a$ and $b$ are two distinct boundary edges of a punctured bordered surface $\mathfrak{S}$. Let $\mathfrak{S}^{\prime}=\mathfrak{S} /(a=b)$, and $c$ be the image of a (or $\left.b\right)$ in $\mathfrak{S}^{\prime}$.

(a) There is a unique $\mathcal{R}$-algebra homomorphism $\rho: \mathscr{S}_{\mathbb{S}}\left(\mathfrak{S}^{\prime}\right) \rightarrow \mathscr{S}_{\mathbf{s}}(\mathfrak{S})$ such that if $\alpha$ is a stated $\partial \mathfrak{S}^{\prime}$-tangle vertically transversal to $c$, then $\rho(\alpha)=\sum_{\beta}[\beta]$, where the sum is over all lifts $\beta$ of $\alpha$, and $[\beta]$ is the element in $\mathscr{S}_{\mathbf{s}}(\mathfrak{S})$ represented by $\beta$.

(b) In addition, $\rho$ is injective. 
(c) For 4 distinct boundary edges $a_{1}, a_{2}, b_{1}, b_{2}$ of $\mathfrak{S}$, the following diagram is commutative:

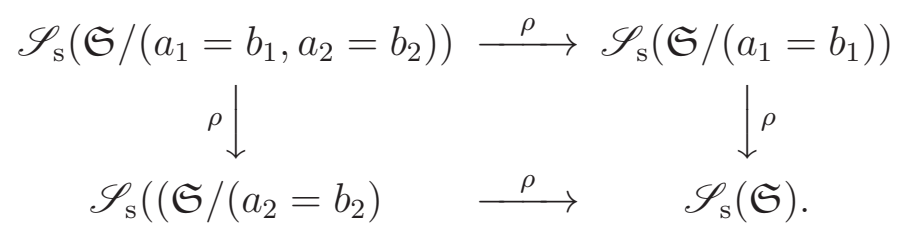

Proof. (a) Let $T(c)$ be the set of all stated $\partial \mathfrak{S}^{\prime}$-tangles vertically transverse to $c$ (no isotopy is considered here), and $V$ be the set of all isotopy classes of stated $\partial \mathfrak{S}^{\prime}$-tangles. The map iso : $T(c) \rightarrow V$, sending an element in $T(c)$ to its isotopy class as a stated $\partial \mathfrak{S}^{\prime}$-tangle, is surjective. Define

$$
\tilde{\rho}: T(c) \rightarrow \mathscr{S}_{\mathrm{S}}(\mathfrak{S}), \quad \tilde{\rho}(\alpha)=\sum_{\beta: \text { lifts of } \alpha}[\beta]
$$

Claim 1. If $\alpha, \alpha^{\prime} \in T(c)$ and $\operatorname{iso}(\alpha)=\operatorname{iso}\left(\alpha^{\prime}\right)$, then $\tilde{\rho}(\alpha)=\tilde{\rho}\left(\alpha^{\prime}\right)$.

Suppose the claim holds. Then $\tilde{\rho}$ descends to a map $\rho^{\prime}: V \rightarrow \mathscr{S}_{\mathrm{s}}(\mathfrak{S})$. Recall $\mathscr{S}_{\mathrm{S}}\left(\mathfrak{S}^{\prime}\right)$ is defined as the $\mathcal{R}$-span of $V$ modulo the defining relations (10) $-(13)$. The locality (of these defining relations) shows that $\rho^{\prime}$ respects the defining relations. Hence $\rho^{\prime}$ descends to an $\mathcal{R}$-homomorphism $\rho: \mathscr{S}_{\mathbf{S}}\left(\mathfrak{S}^{\prime}\right) \rightarrow \mathscr{S}_{\mathrm{S}}(\mathfrak{S})$, which is clearly an $\mathcal{R}$-algebra homomorphism.

It remains to prove Claim 1 . We break the proof into steps.

Step 1. Let $\mathcal{D}(c)$ be the set of all stated $\partial \mathfrak{S}^{\prime}$-tangle diagrams transversal to $c$. Each $D \in \mathcal{D}(c)$ is equipped with the positive boundary order. For a total order $\mathcal{O}$ on $D \cap c$ and a map $\varepsilon: D \cap c \rightarrow\{ \pm\}$ let $\tilde{D}(\mathcal{O}, \boldsymbol{\varepsilon})$ be the stated $\partial \mathfrak{S}$-tangle diagram obtained from $D$ by splitting along $c$. Here the height order and the states on $a$ and $b$ are the lifts of $\mathcal{O}$ and $\varepsilon$, while the height order and the states on other boundary edges are the lifts of the corresponding ones of $D$. Define

$$
\tilde{\rho}(D, \mathcal{O})=\sum_{\varepsilon} \tilde{D}(\mathcal{O}, \boldsymbol{\varepsilon}), \quad \text { sum is over all maps } \varepsilon: D \cap c \rightarrow\{ \pm\} .
$$

Step 2. Recall that $\alpha \in T(c)$, i.e. $\alpha$ is a stated $\partial \mathfrak{S}^{\prime}$-tangle vertically transversal to $c$. A small smooth isotopy, keeping framing vertical on $c \times(0,1)$, does not move $\alpha$ out of $T(c)$, and does not change $\tilde{\rho}(\alpha)$. Thus, after a small smooth isotopy of this type we can assume that $\alpha$ has a stated $\partial \mathfrak{S}^{\prime}$-tangle diagram $D \in \mathcal{D}(c)$. The height order on $\alpha \cap c \times(0,1)$ induces a total order $\mathcal{O}$ on $D \cap c$. From the definition, $\tilde{\rho}(\alpha)=\tilde{\rho}(D, \mathcal{O})$.

Similarly, $\alpha^{\prime}$ is presented by $\left(D^{\prime}, \mathcal{O}^{\prime}\right)$, where $D^{\prime} \in \mathcal{D}(c)$ and $\mathcal{O}^{\prime}$ is a total order on $D^{\prime} \cap c$. To prove the claim, we need to show that $\tilde{\rho}(D, \mathcal{O})=\tilde{\rho}\left(D^{\prime}, \mathcal{O}^{\prime}\right)$.

Step 3. Recall that $\alpha$ and $\alpha^{\prime}$ are isotopic and their diagrams $D, D^{\prime}$ are transersal to $c$. By considering Reidemeister moves involving $D \cup c$, we see that $D^{\prime} \cup c$ can be obtained from $D \cup c$ by a sequence of moves, each is

(i) a Reidemeister move RI, RII, or RIII not involving $c$; or

(ii) move IIa (which involves $c$ ) as shown in Figure 6: or

(iii) move IIIa (which involves $c$ ) as shown in Figure 6; or

(iv) move IV which reorders the total order on $D \cap c$, see Figure 6;

or an isotopy of $\mathfrak{S}^{\prime}$ which fixes $c$ as a set during the isotopy. 


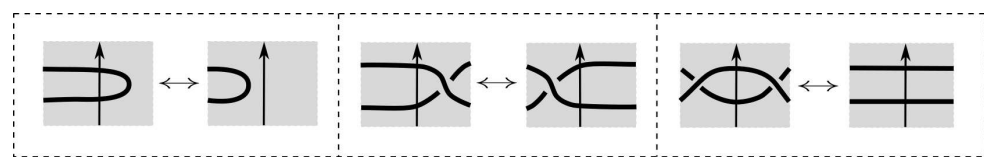

Figure 6. Move Ila (left), Move IIla (middle), and Move IV (right). The vertical line is part of $c$, and the arrow indicates the order.

It is clear that $\tilde{\rho}$ is invariant under isotopy of $\mathfrak{S}^{\prime}$ which fixes $c$ and moves RI, RII, and RIII not involving $c$. We will see that if $\tilde{\rho}$ is invariant under move IIa, then it is invariant under all other moves.

Step 4. Now we show that $\tilde{\rho}$ is invariant under move IIa. We have

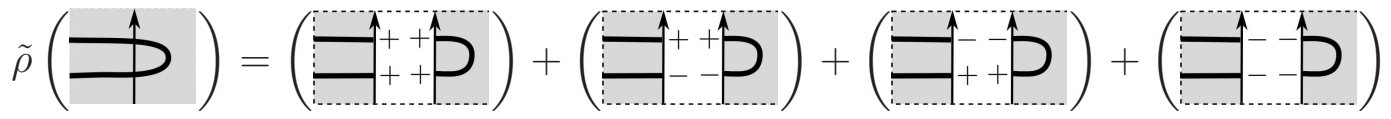

$$
\begin{aligned}
& =-q^{5 / 2}\left(\begin{array}{l}
+ \\
-
\end{array}\right]+q^{1 / 2}\left(\begin{array}{c}
-\uparrow \\
+
\end{array}\right)
\end{aligned}
$$

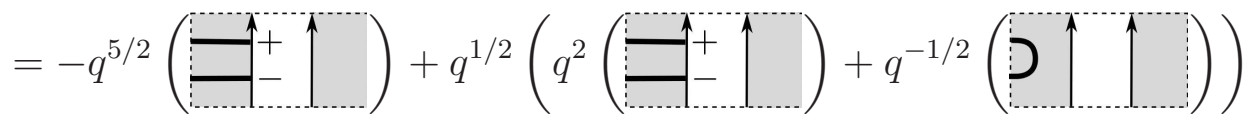

$$
\begin{aligned}
& =\left(\Gamma^{\uparrow} \uparrow\right)=\tilde{\rho}(\supset \uparrow) \text {. }
\end{aligned}
$$

where the second identity follows from the values of trivial arcs given by (12) and Lemma 2.3, while the third identity follows from (13). Thus, $\tilde{\rho}$ is invariant under Move IIa.

Step 5. Now we show that the invariance of Moves IIIa and IV follows from the invariance of Moves RI, RII, RIII (not involving c), and IIa. Consider IIIa. Using the skein relation, we have

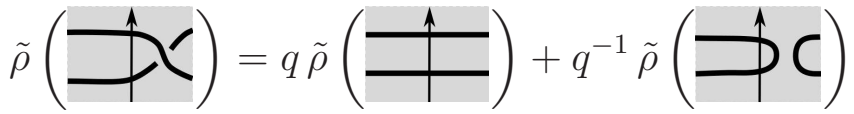

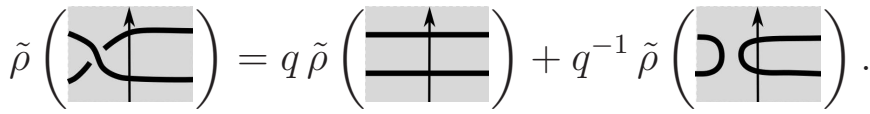

The right hand sides of (27) and (28) are equal by move IIa, proving the invariance of IIIa.

Finally consider Move IV. Using the skein relation at the two crossings, we have

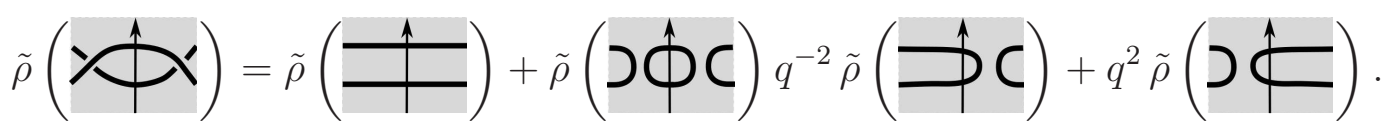

Using Move IIa in the last 3 terms of the right hand side, then the trivial loop relation which says a trivial knot is $-q^{2}-q^{-2}$, we get

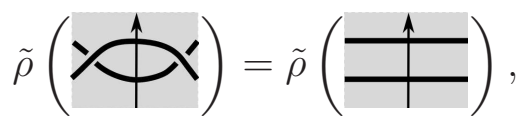

which proves that $\tilde{\rho}$ is invariant under Move IV. This completes the proof of part (a). 
(b) Fix an orientation $\mathfrak{o}^{\prime}$ of $\partial \mathfrak{S}^{\prime}$ and an orientation of $c$. Define the orientation $\mathfrak{o}$ of $\partial \mathfrak{S}$ such that the map pr $: \mathfrak{S} \rightarrow \mathfrak{S}^{\prime}$ preserve the orientation on edge boundary edge of $\mathfrak{S}$. We will equip any $\partial \mathfrak{S}^{\prime}$-tangle diagram (resp. $\partial \mathfrak{S}^{\prime}$-tangle diagram) with the $\mathfrak{o}^{\prime}$-order (resp. o-order).

Suppose $D$ is an increasingly stated $\partial \mathfrak{S}^{\prime}$-tangle diagram transversal to $c$. Let $\tilde{D}(+)$ be the lift of $D$ in which all the state of every endpoint in $a$ (and hence in $b$ ) is + . Note that the state of $\tilde{D}(+)$ is also increasing. In general, $\tilde{D}$ may not be simple. After an isotopy we can assume that $D$ is $c$-normal, i.e. $|D \cap c|=\mu(D, c)$, which is the smallest integer among all $\left|D^{\prime} \cap c\right|$ with $D^{\prime}$ isotopic to $D$. Then $\tilde{D}=\operatorname{pr}^{-1}(D)$ is a simple $\partial \mathfrak{S}$-tangle diagram, and from the definition of $\rho$ and the $b$-leading term (see Section 2.12), we have

$$
\operatorname{lt}_{a}(\rho(D))=\tilde{D}(+) \text { in } \mathscr{S}_{\mathbf{s}}(\mathfrak{S}) \text {. }
$$

In particular, the isotopy class of $\tilde{D}(+)$ does not depend on how we isotope $D$ to a $c$-normal position. Note that the isotopy class of $\tilde{D}(+)$ totally determine the isotopy class of $D$, i.e. the following map is injective:

$$
B\left(\mathfrak{o}^{\prime} ; \mathfrak{S}^{\prime}\right) \rightarrow B(\mathfrak{o} ; \mathfrak{S}), \quad D \rightarrow \tilde{D}(+)
$$

Suppose $0 \neq x \in \mathscr{S}_{\mathbf{s}}\left(\mathfrak{S}^{\prime}\right)$. Then $x=\sum_{j} c_{j} D_{j}$, where $0 \neq c_{j} \in \mathcal{R}$ and $D_{j} \in B\left(\mathfrak{o}^{\prime} ; \mathfrak{S}^{\prime}\right)$. Assume $\max _{j} \mu\left(D_{j}, c\right)=k$. From (29) and the injectivity of the map (30), we have

$$
\operatorname{lt}_{a}(\rho(x))=\sum_{\mu\left(D_{j}, c\right)=k} c_{j} \tilde{D}_{j}(+) \neq 0 .
$$

This proves $\rho(x) \neq 0$, and $\rho$ is injective.

(c) The commutativity of Diagram (26) follows immediately from the definition.

3.3. Triangular decomposition. Suppose a punctured bordered surface $\mathfrak{S}$ is obtained by removing a finite set $\mathcal{P}$ from a compact oriented surface $\overline{\mathfrak{S}}$.

Suppose $\Delta$ is an ideal triangulation of $\mathfrak{S}$, i.e. a triangulation of $\mathbb{\mathfrak { S }}$ whose vertex set is exactly $\mathcal{P}$. By cutting along all the edges of $\Delta$, we see that there is a finite collection $\tilde{\mathcal{F}}=\tilde{\mathcal{F}}(\Delta)$ of disjoint ideal triangles and a finite collection of disjoint pairs of elements in $\tilde{\mathcal{E}}=\tilde{\mathcal{E}}(\Delta)$, the set of all edges of ideal triangles in $\tilde{\mathcal{F}}$, such that $\mathfrak{S}$ is obtained from $\tilde{\mathfrak{S}}:=\bigsqcup_{\mathfrak{T} \in \tilde{\mathcal{F}}} \mathfrak{T}$ by gluing the two edges in each pair. It may happen that two edges of one triangle are glued together.

From Theorem 3.1 we have an injective algebra homomorphism

$$
\rho_{\Delta}: \mathscr{S}_{\mathrm{S}}(\mathfrak{S}) \hookrightarrow \bigotimes_{\tau \in \mathcal{F}(\Delta)} \mathscr{S}_{\mathrm{S}}(\mathfrak{T})
$$

The map $\rho_{\Delta}$ is described explicitly by Theorem 3.1. It is natural now to study the stated skein algebra of an ideal triangle.

It is known that $\mathfrak{S}$ is triangulable, i.e. it has a triangulation, if and only if $|\mathcal{P}| \geq 1$ and $(\overline{\mathfrak{S}}, \mathcal{P})$ is not one of the followings: (i) $\overline{\mathfrak{S}}$ is a sphere with $|\mathcal{P}| \leq 2$, (ii) $\overline{\mathfrak{S}}$ is a disk with $\mathcal{P} \subset \partial \Sigma$ and $|\mathcal{P}| \leq 2$. 
3.4. On the uniqueness of the defining relations. Suppose we modify the defining relations by replacing (12) and (13) with respectively the more general

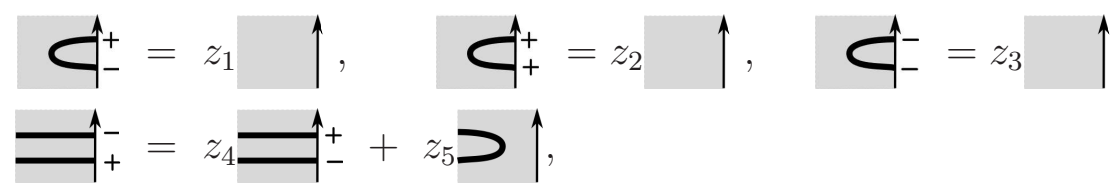

where $z_{i} \in \mathcal{R}$. Then it is easy the set $B(\mathfrak{S})$, still spans the new $\mathscr{S}_{\mathbf{s}}(\mathfrak{S})$. If we want (i) consistency: $B(\mathfrak{S})$ is a basis of $\mathscr{S}_{\mathfrak{S}}(\mathfrak{S})$ and (ii) decomposition: Theorem 3.1 holds, then repeating the proofs we can find exactly 4 solutions $\left(z_{1}, z_{2}, z_{3}, z_{4}, z_{5}\right)$. In all of them $z_{2}=$ $z_{3}=0$. The four solutions are

$$
\begin{aligned}
& z_{1}=z_{5}=\varepsilon q^{-1 / 2}, z_{4}=q^{2}, \quad \varepsilon \in\{ \pm 1\} \\
& z_{1}=z_{5}=\varepsilon q^{-5 / 2}, z_{4}=q^{-2}, \quad \varepsilon \in\{ \pm 1\} .
\end{aligned}
$$

The group $\mathbb{Z} / 2 \times \mathbb{Z} / 2$, generated by two commuting involutions, acts on the set of solutions as follows. The first involution replaces each diagram $\alpha$ with $k+$ states in (33) and (34) by $(-1)^{k} \alpha$. The second involution switches all the states from - to + and + to - in (33) and (34). It is easy to see that each involution transforms a solution to another solution. Then all the 4 solutions are obtained from one of them, say the solution we used in (12) and (13), by the action of this group $\mathbb{Z} / 2 \times \mathbb{Z} / 2$. In this sense our solution is unique.

Remark 3.2. Using Theorem 3.1 one can interpret the assignment $\mathfrak{S} \rightarrow \mathscr{S}_{\mathrm{s}}(\mathfrak{S})$ as a copresentation of a certain modular operad of surfaces. For details of modular operads, see [Vo].

\section{IDEAL BIGON AND IDEAL TRIANGLE}

4.1. Definitions and notations. An $\operatorname{arc} \alpha$ in a punctured bordered surface $\mathfrak{S}$ is a properly embedded submanifold diffeomorphic to $[0,1]$. If the two end points of $\alpha$ are in the same boundary edge, we call $\alpha$ a returning arcs.

Suppose $s: \partial(\alpha) \rightarrow\{ \pm\}$ is a state of a $\partial \mathfrak{S}$-tangle $\alpha$, where $\mathfrak{S}$ is a punctured bordered surface. A permutation of $s$ is any state of the form $s \circ \sigma$, where $\sigma: \partial(\alpha) \rightarrow \partial(\alpha)$ is a bijection such that if $x \in b$, where $b$ is a boundary edge, then $\sigma(x) \in b$. The only permutation of $s$ which is increasing is denoted by $s^{\uparrow}$.

Suppose $s: \partial(\alpha) \rightarrow\{ \pm\}$ is not increasing. There there is a pair $u, v \in \partial(\alpha)$ such that $u>v, u$ and $v$ are consecutive in the height order, and $s(u)=-, s(v)=+$. The new state $s^{\prime}: \partial(\alpha) \rightarrow\{ \pm\}$, which is equal to $s$ everywhere except $s^{\prime}(u)=+, s^{\prime}(v)=-$, is called a simple positive permutation of $s$.

For elements $x, y$ of an $\mathcal{R}$-module, $x \doteq y$ will mean there is an integer $j$ such that $x=q^{j / 2} y$.

4.2. Ideal bigon. Suppose $\mathfrak{B}$ is an ideal bigon, i.e. $\mathfrak{B}$ is obtained from a disk by removing 2 points on its boundary. Let $a$ and $b$ be the boundary edges of $\mathfrak{B}$. Let $\alpha$ be an arc whose two end points are not in the same boundary edge, and let $\alpha(k)$ be $k$ parallels of $\alpha$. See Figure 7 . Unless otherwise stated, the order of each $\partial \mathfrak{B}$-tangle diagram is positive. For example, the diagram of $\alpha^{2}$ is different from $\alpha(2)$ and is depicted in Figure 7 . For $\varepsilon, \varepsilon^{\prime} \in\{ \pm\}$ let $\alpha\left(\varepsilon, \varepsilon^{\prime}\right)$ be $\alpha$ equipped with the state $s$ such that $\left.s(\alpha \cap a)=\varepsilon, s(\alpha \cap b)=\varepsilon^{\prime}\right)$. Recall that $C_{\varepsilon^{\prime}}^{\varepsilon}$ is defined by (15). 


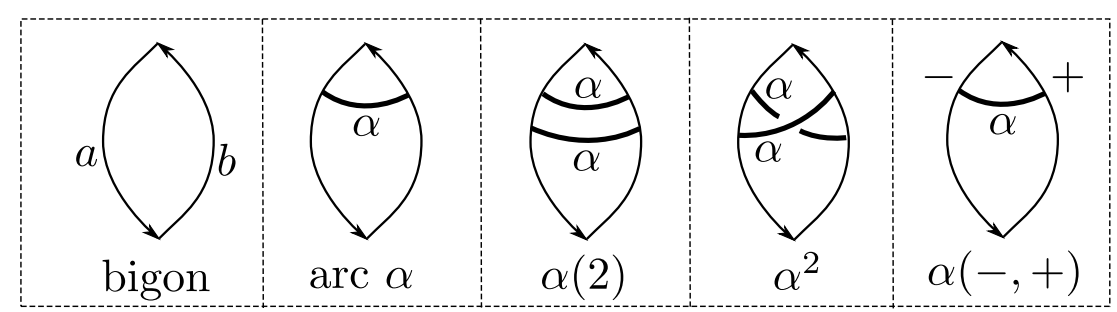

Figure 7. From left to right: bigon, $\operatorname{arc} \alpha, \alpha(2), \alpha^{2}$, and $\alpha(-,+)$

Theorem 4.1. Let $\mathfrak{B}$ be an ideal bigon with the above notations. Then $\mathscr{S}_{\mathrm{s}}(\mathfrak{B})$ is the $\mathcal{R}$ algebra generated by $X=\left\{\alpha\left(\varepsilon, \varepsilon^{\prime}\right) \mid \varepsilon, \varepsilon^{\prime} \in\{ \pm\}\right\}$, subject to the relations

$$
\begin{aligned}
& \alpha(\varepsilon,-) \alpha\left(\varepsilon^{\prime},+\right)=q^{2} \alpha(\varepsilon,+) \alpha\left(\varepsilon^{\prime},-\right)-q^{5 / 2} C_{\varepsilon^{\prime}}^{\varepsilon} \quad \forall \varepsilon, \varepsilon^{\prime} \in\{ \pm\} \\
& \alpha(-, \varepsilon) \alpha\left(+, \varepsilon^{\prime}\right)=q^{2} \alpha(+, \varepsilon) \alpha\left(-, \varepsilon^{\prime}\right)-q^{5 / 2} C_{\varepsilon^{\prime}}^{\varepsilon} \quad \forall \varepsilon, \varepsilon^{\prime} \in\{ \pm\} .
\end{aligned}
$$

Remark 4.2. If $\tau_{2}$ is the rotation by $\pi$ about the center of $\mathfrak{B}$, so that $\tau_{2}(a)=b, \tau_{2}(b)=a$, then (36) is the image of (35) under $\tau_{2}$.

Proof. The proof is simple, but we want to give all details here, since we will use a similar proof for the case of an ideal triangle later. The first, and easy, step is to show that $\mathscr{S}_{\mathrm{s}}(\mathfrak{B})$ is generated by $X$ and the relations (35)-(36) are satisfied. Then, since we know an explicit $\mathcal{R}$-basis of $\mathscr{S}_{\mathrm{S}}(\mathfrak{S})$, an upper estimate argument will finish the proof.

Step 1. For each $k \in \mathbb{N}$, the set $\partial(k):=\partial(\alpha(k))$ consists of $k$ points in $a$ and $k$ points in $b$. Let $\operatorname{St}(k)$ be the set of all states $s: \partial(k) \rightarrow\{ \pm\}$, and $\operatorname{St}^{\uparrow}(k) \subset \operatorname{St}(k)$ be the subset of all increasing states. For $s \in \operatorname{St}(k)$ let $\alpha(k, s)$ be the stated $\partial \mathfrak{B}$-tangle diagram, which is $\alpha(k)$ equipped with state $s$. Similarly, $\left(\alpha^{k}, s\right)$ is $\alpha^{k}$ equipped with $s$. Recall that we have an increasing filtration $\left\{F_{m}\left(\mathscr{S}_{\mathrm{s}}(\mathfrak{B})\right)\right\}$ of $\mathscr{S}_{\mathrm{s}}(\mathfrak{B})$ and its associated graded algebra $\operatorname{Gr}\left(\mathscr{S}_{\mathrm{s}}(\mathfrak{B})\right)$. By Proposition 2.12, the set $\left\{\alpha(m, s) \mid s \in \mathrm{St}^{\uparrow}(m)\right\}$ is an $\mathcal{R}$-basis of $\operatorname{Gr}_{m}\left(\mathscr{S}_{\mathrm{S}}(\mathfrak{B})\right)$.

By Lemma 4.3, $\left(\alpha^{m}, s\right) \doteq \alpha(m, s)\left(\bmod F_{m-1}\left(\mathscr{S}_{\mathrm{s}}(\mathfrak{B})\right)\right)$, which shows that

$$
B_{m}:=\left\{\left(\alpha^{m}, s\right) \mid s \in \operatorname{St}^{\uparrow}(m)\right\}
$$

is also an $\mathcal{R}$-basis of $\operatorname{Gr}_{m}\left(\mathscr{S}_{\mathrm{S}}(\mathfrak{B})\right)$. It follows that $\left\{\left(\alpha^{k}, s\right) \mid k \in \mathbb{N}, s \in \mathrm{St}^{\uparrow}(k)\right\}$ is an $\mathcal{R}$-basis of $\mathscr{S}_{\mathrm{S}}(\mathfrak{B})$. Since $\left(\alpha^{k}, s\right)$ is a monomial in the letters in $X$, we conclude that $X$ generates $\mathscr{S}_{\mathrm{s}}(\mathfrak{B})$ as an $\mathcal{R}$-algebra.

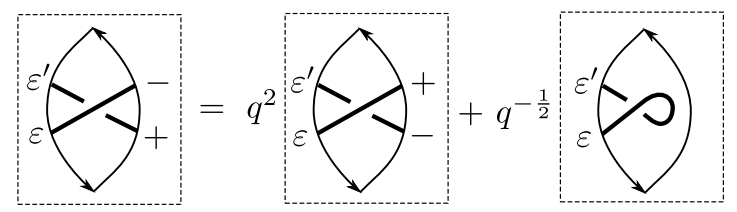

Figure 8. An application of Relation (13)

Let us now prove (35). Apply (13) as in Figure 8, then use (16) to remove the kink; we get

$$
\alpha(\varepsilon,-) \alpha\left(\varepsilon^{\prime},+\right)=q^{2} \alpha(\varepsilon,+) \alpha\left(\varepsilon^{\prime},-\right)+q^{-1 / 2}\left(-q^{3}\right) C_{\varepsilon^{\prime}}^{\varepsilon},
$$

which is (35). The proof of (36) is similar. 
Step 2. Let $A$ be the $\mathcal{R}$-algebra generated by $X$ subject to the relations (35) and (36)). Then $A$ is a filtered $\mathcal{R}$-algebra, where the $m$-th filtration $F_{m}(A)$ is spanned by the set of monomials in $X$ of degree $\leq m$. The $\mathcal{R}$-algebra map $\omega: A \rightarrow \mathscr{S}_{\mathrm{s}}(\mathfrak{B})$ defined by $\omega(x)=x$ for all $x \in X$, is a surjective homomorphism of filtered algebras, and induces an algebra homomorphism $\operatorname{Gr}(\omega): \operatorname{Gr}(A) \rightarrow \operatorname{Gr}\left(\mathscr{S}_{\mathbf{s}}(\mathfrak{B})\right)$. The set $M_{m}:=\left\{\vartheta_{1} \ldots \vartheta_{m} \mid \vartheta_{j} \in X\right\}$ spans $\operatorname{Gr}_{m}(A)$. Presenting each $\vartheta_{j}$ as a stated arc on $\mathfrak{B}$, we see that there is state $s \in \operatorname{St}(m)$ such that $\vartheta_{1} \ldots \vartheta_{m}=\left(\alpha^{m}, s\right)$, and we use this to identify $M_{m}$ with the set $\left\{\left(\alpha^{m}, s\right) \mid s \in \operatorname{St}(m)\right\}$.

Step 3. Since the second term on the right hand side of (35) has degree less than other terms, in $\operatorname{Gr}(A)$ we have relation (35), with the second term of the right hand side removed:

$$
\alpha(\varepsilon,-) \alpha\left(\varepsilon^{\prime},+\right)=q^{2} \alpha(\varepsilon,+) \alpha\left(\varepsilon^{\prime},-\right) .
$$

There are states $r, r^{\prime} \in \mathrm{St}(2)$ such that the left hand side and the right hand side of the above are respectively $\left(\alpha^{2}, r\right)$ and $\left(\alpha^{2}, r^{\prime}\right)$, and the above relation can be rewritten as

$$
\left(\alpha^{2}, r\right)=q^{2}\left(\alpha^{2}, r^{\prime}\right) \text { in } \operatorname{Gr}(A) \text {. }
$$

The upshot is that $r^{\prime}$ is a simple positive permutation of $r$, see Figure 8 ,

Step 4. Let us show that the subset $M^{\uparrow}(m):=\left\{\left(\alpha^{m}, s\right) \mid s \in \operatorname{St}^{\uparrow}(m)\right\}$ spans $\operatorname{Gr}_{m}(A)$. Suppose $\vartheta_{1} \ldots \vartheta_{m}=\left(\alpha^{m}, s\right) \in M(m)$ with $\mathrm{nd}(s)>0$. Then there is a consecutive $s$ decreasing pair $(u, v) \in \partial(m)^{2}$. Both $u, v$ belong to the same boundary edge, say $b$. Assume $u$ is an end point of $\vartheta_{j}$, then $v$ must be an end point of $\vartheta_{j+1}$. Then $\vartheta_{j} \vartheta_{j+1}$ look like in the left hand side of Figure 8, i.e. $\vartheta_{j} \vartheta_{j+1}$ is exactly the left hand side of (35), or the left hand side of (37). Replacing $\vartheta_{j} \vartheta_{j+1}$ by the right hand side of (37), we get

$$
\left(\alpha^{m}, s\right) \doteq\left(\alpha^{m}, s^{\prime}\right) \quad\left(\bmod F_{m-1}(A)\right),
$$

where $s^{\prime}$ is a simple positive permutation of $s$. An induction on $\operatorname{nd}(s)$ shows that for any $s \in \operatorname{St}(m)$, we have

$$
\left(\alpha^{m}, s\right) \doteq\left(\alpha, s^{\uparrow}\right) \quad\left(\bmod F_{m-1}(A)\right),
$$

which, in turns, shows that $M_{m}^{\uparrow}$ also spans $\operatorname{Gr}_{m}(A)$.

Since $\operatorname{Gr}(\omega)\left(M_{m}^{\uparrow}\right)=B_{m}$, which is an $\mathcal{R}$-basis of $\operatorname{Gr}_{m}\left(\mathscr{S}_{\mathrm{s}}(\mathfrak{B})\right), M_{m}^{\uparrow}$ is $\mathcal{R}$-linearly independent. Thus, $M_{m}^{\uparrow}$ is an $\mathcal{R}$-basis of $\operatorname{Gr}_{m}(A)$, and $\operatorname{Gr}(\omega): \operatorname{Gr}_{m}(A) \rightarrow \operatorname{Gr}_{m}\left(\mathscr{S}_{\mathrm{s}}(\mathfrak{B})\right)$ is an isomorphism. It follows that $\omega: A \rightarrow \mathscr{S}_{\mathrm{s}}(\mathfrak{B})$ is an isomorphism.

The following lemma is used in the proof of Theorem 4.1, and we use notations there.

Lemma 4.3. Suppose $D$ is a stated $\partial \mathfrak{B}$-tangle diagram with $\partial D=\partial(k)$ and each component of $D$ is an arc. Let $s \in \mathrm{St}(k)$ be the state of $D$.

(a) If $D$ contains a returning arc, then, as an element in $\mathscr{S}_{\mathrm{s}}(\mathfrak{B}), D \in F_{k-1}\left(\mathscr{S}_{\mathrm{s}}(\mathfrak{B})\right)$.

(b) If $D$ has no returning arcs, then, as elements in $\mathscr{S}_{\mathrm{s}}(\mathfrak{B})$,

$$
D \doteq \alpha(k, s) \quad\left(\bmod F_{k-1}\left(\mathscr{S}_{\mathrm{s}}(\mathfrak{B})\right)\right) .
$$

Proof. (a) If there is no double point on a returning arc, then $D \in F_{k-1}\left(\mathscr{S}_{\mathrm{s}}(\mathfrak{B})\right)$ by relation (17). Suppose there is a double point on a returning arc. Each of the two smooth resolutions of this double point contains a returning arc, see Figure 9. The skein relation and induction show that $D \in F_{k-1}\left(\mathscr{S}_{\mathrm{s}}(\mathfrak{B})\right)$. 


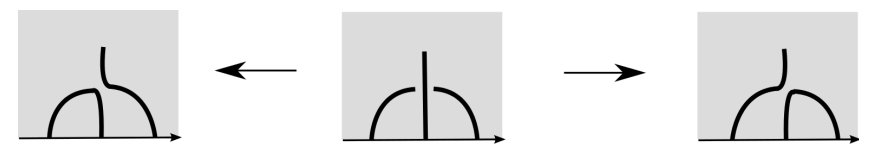

Figure 9. A returning arc with a double point on it (middle), and two of its resolutions (left and right)

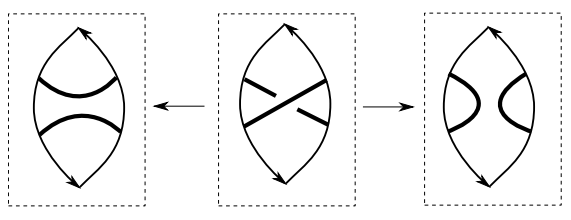

Figure 10. A double point of 2 non-returning arcs (middle) and its two resolutions (left and right)

(b) If $D$ has no double point, then $D=\alpha(k, s)$. Suppose $D$ has a double point. Of the two resolutions of the double point, exactly one does not have a returning arc; see Figure 18 , By the skein relation, part (a), and induction, we have $D \doteq \alpha(k, s)\left(\bmod F_{k-1}\right)$.

Proposition 4.4. Suppose $\mathcal{R}$ is a domain. Then $\mathscr{S}_{\mathrm{s}}(\mathfrak{B})$ is a domain.

Proof. We say an $\mathcal{R}$-basis $\left\{b_{i} \mid i \in I\right\}$ of an $\mathcal{R}$-algebra $A$ is compatibly ordered, if $I$ is a monoid equipped with a total order such that if $i \leq i^{\prime}$ and $j \leq j^{\prime}$ then $i+j \leq i^{\prime}+j^{\prime}$, and $b_{i} b_{j} \doteq b_{i+j}$. We first proved the following lemma.

Lemma 4.5. Suppose $\mathcal{R}$ is a commutative domain and an $\mathcal{R}$-algebra $A$ has a compatibly ordered basis. Then $A$ is a domain.

Proof. Suppose $x \in A$ is non-zero. Then $x=\sum_{i} x_{i} b_{i} \in A$, with $c_{i} \in \mathcal{R}$. The leading term $\operatorname{lt}(x)$ is defined to be $c_{j} b_{j}$, where $j$ is the largest index such that $c_{j} \neq 0$. Suppose $y \neq 0$ and $\operatorname{lt}(y)=c_{l}^{\prime} b_{l}$. From the assumptions $\operatorname{lt}(x y) \doteq c_{j} c_{l}^{\prime} b_{j+l} \neq 0$.

Return to the proof of the proposition. Let $I \subset \mathbb{N}^{3}$ be the set of all $\mathbf{k}=\left(k, k_{a}, k_{b}\right) \in \mathbb{N}^{3}$ such that $k_{a}, k_{b} \leq k$. For $\mathbf{k} \in Q$ define $b_{\mathbf{k}}=\left(\alpha^{k}, s\right)$, where $s \in \operatorname{St}^{\uparrow}(k)$ is the only increasing state which has $k_{a}$ pluses on edge $a$ and $k_{b}$ pluses on edge $b$. Then $\left\{b_{\mathbf{k}} \mid \mathbf{k} \in I\right\}$ is an $\mathcal{R}$-basis of $\mathscr{S}_{\mathrm{s}}(\mathfrak{B})$. Order $I$ lexicographically. Lemma 4.3 shows that

$$
z(\mathbf{k}) z\left(\mathbf{k}^{\prime}\right) \doteq z\left(\mathbf{k}+\mathbf{k}^{\prime}\right) \quad \text { in } \operatorname{Gr}\left(\mathscr{S}_{\mathbf{s}}(\mathfrak{B})\right)
$$

In other words, $\left\{b_{\mathbf{k}} \mid \mathbf{k} \in Q\right\}$ is a compatibly ordered basis of $\operatorname{Gr}\left(\mathscr{S}_{\mathrm{s}}(\mathfrak{B})\right)$. By the lemma, $\operatorname{Gr}\left(\mathscr{S}_{\mathrm{s}}(\mathfrak{B})\right)$ is a domain. Hence, $\mathscr{S}_{\mathrm{s}}(\mathfrak{B})$ is a domain.

4.3. Ideal triangle. Let $\mathfrak{T}$ be an ideal triangle, with boundary edges $a, b, c$ and $\operatorname{arcs} \alpha, \beta, \gamma$ in counterclockwise order, as in Figure 11.

Let $\tau$ be the counterclockwise rotation by $2 \pi / 3$, so that $\tau(\mathfrak{T})=\mathfrak{T}$ and $\tau$ gives the cyclic permutation $a \rightarrow b \rightarrow c \rightarrow a$ and $\alpha \rightarrow \beta \rightarrow \gamma \rightarrow \alpha$. For $\varepsilon, \varepsilon^{\prime} \in\{ \pm\}$, let $\alpha\left(\varepsilon, \varepsilon^{\prime}\right)$ be $\alpha$ with and the state $s$ given by $s(\alpha \cap c)=\varepsilon, s(\alpha \cap b)=\varepsilon^{\prime}$. Let $\beta\left(\varepsilon, \varepsilon^{\prime}\right)=\tau\left(\alpha\left(\varepsilon, \varepsilon^{\prime}\right)\right)$ and $\gamma\left(\varepsilon, \varepsilon^{\prime}\right)=\tau^{2}\left(\alpha\left(\varepsilon, \varepsilon^{\prime}\right)\right)$. Note that $\tau$ defines an automorphism of the algebra $\mathscr{S}_{\mathrm{s}}(\mathfrak{T})$. 


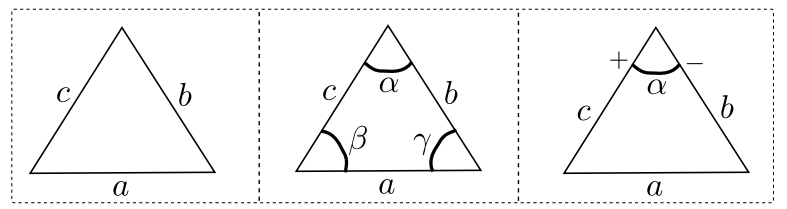

Figure 11. Ideal triangle $\mathfrak{T}$ (left), with $\operatorname{arcs} \alpha, \beta, \gamma$ (middle), and $\alpha(+-)$ (right).

Theorem 4.6. Suppose $\mathfrak{T}$ is an ideal triangle, with the above notations. Then $\mathscr{S}_{\mathrm{s}}(\mathfrak{T})$ is the $\mathcal{R}$-algebra generated by the set of twelve generators

$$
X=\left\{\alpha\left(\varepsilon, \varepsilon^{\prime}\right), \beta\left(\varepsilon, \varepsilon^{\prime}\right), \gamma\left(\varepsilon, \varepsilon^{\prime}\right) \mid \varepsilon, \varepsilon^{\prime} \in\{ \pm\}\right\}
$$

subject to the the following relations and their images under $\tau$ and $\tau^{2}$ :

$$
\begin{aligned}
& \beta(\mu, \varepsilon) \alpha\left(\mu^{\prime}, \varepsilon^{\prime}\right)=q \alpha\left(\varepsilon, \varepsilon^{\prime}\right) \beta\left(\mu, \mu^{\prime}\right)-q^{2} C_{\mu^{\prime}}^{\varepsilon} \gamma\left(\varepsilon^{\prime}, \mu\right) \\
& \alpha(-, \varepsilon) \alpha\left(+, \varepsilon^{\prime}\right)=q^{2} \alpha(+, \varepsilon) \alpha\left(-, \varepsilon^{\prime}\right)-q^{5 / 2} C_{\varepsilon^{\prime}}^{\varepsilon} \\
& \alpha(\varepsilon,-) \alpha\left(\varepsilon^{\prime},+\right)=q^{2} \alpha(\varepsilon,+) \alpha\left(\varepsilon^{\prime},-\right)-q^{5 / 2} C_{\varepsilon^{\prime}}^{\varepsilon} \\
& \alpha(-, \varepsilon) \beta\left(\varepsilon^{\prime},+\right)=q^{2} \alpha(+, \varepsilon) \beta\left(\varepsilon^{\prime},-\right)-q^{5 / 2} \gamma\left(\varepsilon, \varepsilon^{\prime}\right) \\
& \alpha(\varepsilon,-) \gamma\left(+, \varepsilon^{\prime}\right)=q^{2} \alpha(\varepsilon,+) \gamma\left(-, \varepsilon^{\prime}\right)+q^{-1 / 2} \beta\left(\varepsilon^{\prime}, \varepsilon\right) .
\end{aligned}
$$

Proof. First we show that $X$ generates $\mathscr{S}_{\mathrm{s}}(\mathfrak{T})$ and all the relations (41)-(45) are satisfied. Then an upper bound estimate argument will finish the proof. Throughout the proof, the

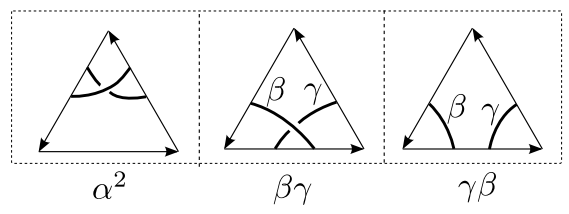

Figure 12. Diagrams of $\alpha^{2}, \beta \gamma$, and $\gamma \beta$

order of each $\partial \mathfrak{T}$-diagram is positive. For example, the diagrams of $\alpha^{2}, \beta \gamma$, and $\gamma \beta$ are depicted in Figure 12 .

Step 1. Let us show that $X$ generates $\mathscr{S}_{\mathrm{s}}(\mathfrak{T})$. For $\mathbf{k}=\left(k_{1}, k_{2}, k_{3}\right) \in \mathbb{N}^{3}$ let $|\mathbf{k}|:=$ $k_{1}+k_{2}+k_{3}$. Let $\theta(\mathbf{k})$ be the simple $\partial \mathfrak{T}$-tangle diagram which consists of $k_{1}$ parallels of $\alpha$, $k_{2}$ parallels of $\beta$, and $k_{3}$ parallels of $\gamma$, and $\theta^{\mathbf{k}}=\alpha^{k_{1}} \beta^{k_{2}} \gamma^{k_{3}}$, see Figure 13.
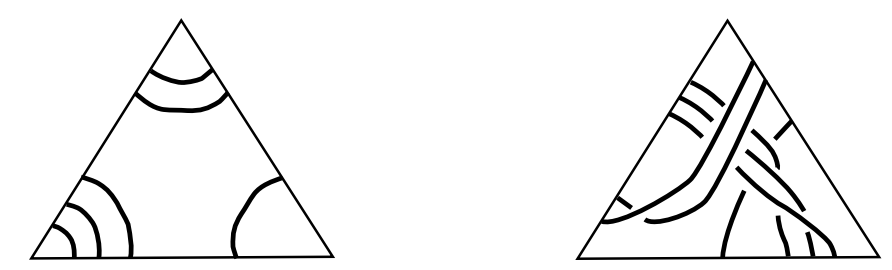

Figure 13. Diagram $\theta(2,3,1)$ (left) and diagram $\alpha^{2} \beta^{3} \gamma$ (right)

The set $\partial(\mathbf{k}):=\partial(\theta(\mathbf{k}))=\partial\left(\theta^{\mathbf{k}}\right)$, considered up to isotopy of $\mathfrak{T}$, consists of $k_{2}+k_{3}$ points on $a, k_{1}+k_{3}$ points on $b$, and $k_{1}+k_{2}$ points on $c$. Let $\operatorname{St}(\mathbf{k})$ be the set of all states 
$s: \partial(\mathbf{k}) \rightarrow\{ \pm\}$, and $\mathrm{St}^{\uparrow}(\mathbf{k}) \subset \mathrm{St}(\mathbf{k})$ be the subset of all increasing states. For $s \in \operatorname{St}(\mathbf{k})$ let $\theta(\mathbf{k}, s)$ be $\theta(\mathbf{k})$ with state $s$. Similarly, $\left(\theta^{\mathbf{k}}, s\right)$ is $\theta^{\mathbf{k}}$ with state $s$. By Proposition 2.12,

$$
B_{m}:=\left\{\theta(\mathbf{k}, s)\left|\mathbf{k} \in \mathbb{N}^{3},\right| \mathbf{k} \mid=m, s \in \mathrm{St}^{\uparrow}(\mathbf{k})\right\}
$$

is an $\mathcal{R}$-basis of $\operatorname{Gr}_{m}\left(\mathscr{S}_{\mathrm{s}}(\mathfrak{T})\right)$. By Lemma 4.7,

$$
\theta(\mathbf{k}, s) \doteq\left(\theta^{\mathbf{k}}, s\right) \quad\left(\bmod F_{|\mathbf{k}|-1}\left(\mathscr{S}_{\mathrm{s}}(\mathfrak{T})\right)\right),
$$

which implies that $\left\{\left(\theta^{\mathbf{k}}, s\right) \mid \mathbf{k} \in \mathbb{N}^{3}, s \in \mathrm{St}^{\uparrow}(\mathbf{k})\right\}$ is also an $\mathcal{R}$-basis of $\mathscr{S}_{\mathbf{s}}(\mathfrak{T})$. Since each $\left(\theta^{\mathbf{k}}, s\right)$ is a monomial in $X, X$ generates $\mathscr{S}_{\mathbf{s}}(\mathfrak{T})$.

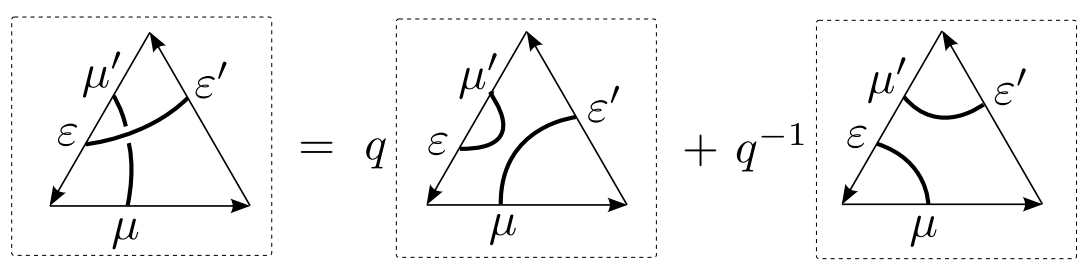

Figure 14. Proof of Identity (46)

Let now prove all the relations (41)-(45) are satisfied. Consider (41). Using the skein relation as in Figure 14, we have

$$
\alpha\left(\varepsilon, \varepsilon^{\prime}\right) \beta\left(\mu, \mu^{\prime}\right)=q C_{\mu^{\prime}}^{\varepsilon} \gamma\left(\varepsilon^{\prime}, \mu\right)+q^{-1} \beta(\mu, \varepsilon) \alpha\left(\mu^{\prime}, \varepsilon^{\prime}\right)
$$

which proves (41).

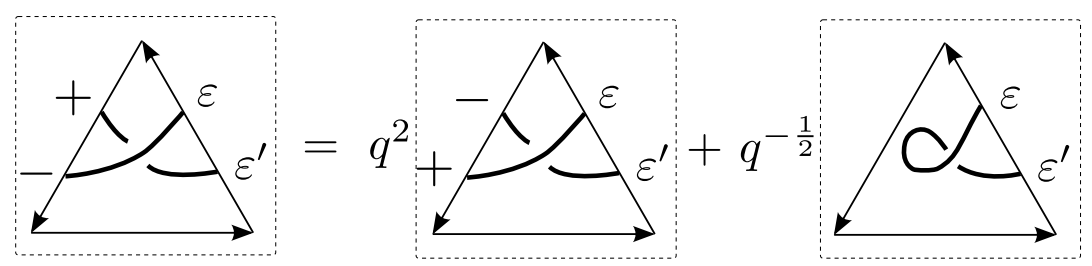

Figure 15. Proof of Identity (47)

Now prove (42). Using Relation (13) as in Figure 15 and then (16), we have

$$
\alpha(-, \varepsilon) \alpha\left(+, \varepsilon^{\prime}\right)=q^{2} \alpha(+, \varepsilon) \alpha\left(-, \varepsilon^{\prime}\right)+q^{1 / 2}\left(-q^{3}\right) C_{\varepsilon^{\prime}}^{\varepsilon},
$$

which proves (42). The proof of (43)-(45) are similar.

Step 2. Let $A$ be the $\mathcal{R}$-algebra generated by $X$ subject to the relations (41)-(45). Then $A$ is a filtered $\mathcal{R}$-algebra where the $m$-th filtration $F_{m}(A)$ is spanned by the set of monomials in $X$ of degree $\leq m$. The $\mathcal{R}$-algebra map $\omega: A \rightarrow \mathscr{S}_{\mathrm{s}}(\mathfrak{B})$, defined by $\omega(x)=x$ for all $x \in X$, is a surjective homomorphism of filtered algebras, and induces an algebra homomorphism

$$
\operatorname{Gr}(\omega): \operatorname{Gr}(A) \rightarrow \operatorname{Gr}\left(\mathscr{S}_{\mathrm{s}}(\mathfrak{B})\right) \text {. }
$$

The set $M_{m}:=\left\{\vartheta_{1} \ldots \vartheta_{m} \mid \vartheta_{j} \in X\right\}$ spans $\operatorname{Gr}_{m}(A)$. If $\alpha_{i} \in\{\alpha, \beta, \gamma\}$ is $\vartheta_{i}$ without state, then by presenting each $\vartheta_{j}$ as a stated arc on $\mathfrak{B}$, there is a state $s: \partial\left(\alpha_{1} \ldots \alpha_{m}\right) \rightarrow\{ \pm\}$ such that $\vartheta_{1} \ldots \vartheta_{m}=\left(\alpha_{1} \ldots \alpha_{m}, s\right)$. Thus, we can identity

$$
M_{m}=\left\{\left(\alpha_{1} \ldots \alpha_{m}, s\right) \mid \alpha_{i} \in\{\alpha, \beta, \gamma\}, s: \partial\left(\alpha_{1} \ldots \alpha_{m}\right) \rightarrow\{ \pm\}\right\} .
$$


Step 3. Let us now show that the subset $\vec{M}_{m} \subset M_{m}$, defined by

$$
\vec{M}_{m}:=\left\{\left(\theta^{\mathbf{k}}, s\right)|| \mathbf{k} \mid=m, s \in \operatorname{St}(\mathbf{k})\right\},
$$

spans $\operatorname{Gr}_{m}(A)$. Ignoring the second term of the right hand side of Relation (41) which is of less degree, we get that for any state $r$ of $\alpha \beta$,

$$
(\beta \alpha, r)=q(\alpha \beta, r) \text { in } \operatorname{Gr}(A) .
$$

Together with its images under $\tau$ and $\tau^{2}$, (48) shows that for any permutation $\sigma$ of $\{1, \ldots, m\}$,

$$
\left(\alpha_{1} \ldots \alpha_{m}, s\right) \doteq\left(\alpha_{\sigma(1)} \ldots \alpha_{\sigma(m)}, s\right) \quad \text { in } \operatorname{Gr}(A) .
$$

In particular, if the numbers of $\alpha, \beta, \gamma$ among $\alpha_{1}, \ldots, \alpha_{m}$ are components of $\mathbf{k}=\left(k_{1}, k_{2}, k_{3}\right)$, then $\left(\alpha_{1} \ldots \alpha_{m}, s\right) \doteq\left(\theta^{\mathbf{k}}, s\right)$. This shows the subset $\vec{M}_{m}$ also spans $\operatorname{Gr}_{m}(A)$.

Step 4. Let us show that the subset $\vec{M}_{m}^{\uparrow} \subset \vec{M}_{m}$, defined by

$$
\vec{M}_{m}^{\uparrow}:=\left\{\left(\theta^{\mathbf{k}}, s\right)|| \mathbf{k} \mid=m, s \in \mathrm{St}^{\uparrow}(\mathbf{k})\right\},
$$

spans $\operatorname{Gr}_{m}(A)$. First we make the following observation. Suppose $\vartheta_{1} \vartheta_{2}$ is the left hand side of one of (42)-(45), and $\bar{\vartheta}_{i} \in\{\alpha, \beta, \gamma\}$ is $\vartheta_{i}$ without states. There is a state $r \in \operatorname{St}\left(\bar{\vartheta}_{1} \bar{\vartheta}_{2}\right)$ such that $\vartheta_{1} \vartheta_{2}=\left(\bar{\vartheta}_{1} \bar{\vartheta}_{2}, r\right)$, and (42)-(45), ignoring the second term of the right hand side, give

$$
\left(\bar{\vartheta}_{1} \bar{\vartheta}_{2}, r\right)=q^{2}\left(\bar{\vartheta}_{1} \bar{\vartheta}_{2}, r^{\prime}\right) \quad \text { in } \operatorname{Gr}(A),
$$

where $r^{\prime}$ is a simple positive permutation of $r$.

Now assume $\vartheta_{1} \ldots \vartheta_{m}=\left(\theta^{\mathbf{k}}, s\right) \in \vec{M}_{m}$ with $\operatorname{nd}(s)>0$. Then there is a consecutive $s$ decreasing pair $(u, v) \in \partial(\mathbf{k})^{2}$. Let $u$ be an end point of $\vartheta_{i}$ and $v$ be an end point of $\vartheta_{j}$. There are two cases: $\bar{\vartheta}_{i}=\bar{\vartheta}_{j}$ and $\bar{\vartheta}_{i} \neq \bar{\vartheta}_{j}$.

Case 1: $\bar{\vartheta}_{i}=\bar{\vartheta}_{j}$. Say, $\bar{\vartheta}_{i}=\bar{\vartheta}_{j}=\alpha$. Then one has $j=i+1$ because $u$ and $v$ are consecutive. Besides, $u, v \in b$ or $u, v \in c$.

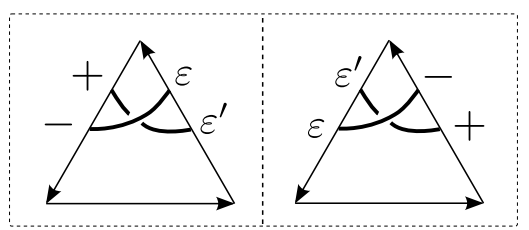

Figure 16. Case 1: we have $\alpha(-, \varepsilon) \alpha\left(+, \varepsilon^{\prime}\right)$ on the left and $\alpha(\varepsilon,-) \alpha\left(\varepsilon^{\prime},+\right)$ on the right

If $u, v \in b$, then $\vartheta_{1} \vartheta_{2}=\alpha(-, \varepsilon) \alpha\left(+, \varepsilon^{\prime}\right)$, the left hand side of (42) (see Figure 16); and if $x_{1}, x_{2} \in c$, then $\vartheta_{1} \vartheta_{2}=\alpha(\varepsilon,-) \alpha\left(\varepsilon^{\prime},+\right)$, the left hand side of (43). Using (50), we get

$$
\left(\theta^{\mathbf{k}}, s\right) \doteq\left(\theta^{\mathbf{k}}, s^{\prime}\right) \quad \text { in } \operatorname{Gr}(A) \text { for some } s^{\prime} \text { with } \operatorname{nd}\left(s^{\prime}\right)<\operatorname{nd}(s) .
$$

Case 2: $\bar{\vartheta}_{i} \neq \bar{\vartheta}_{j}$. There are 3 subcases: (i) $u, v \in c$, (ii) $u, v \in b$, and (iii): $u, v \in a$. See Figure 17.

Subcase 2(i): $u, v \in c$. In this case one has $j=i+1$, and $\vartheta_{i} \vartheta_{i+1}=\alpha(-, \varepsilon) \beta\left(\varepsilon^{\prime},+\right)$, which is the left hand side of (42) (see Figure 17). Again, using (50), we get (51).

Subcase 2(iii) is similar. Actually applying the rotation $\tau$, one gets subcase 2(iii) from 2(i). 


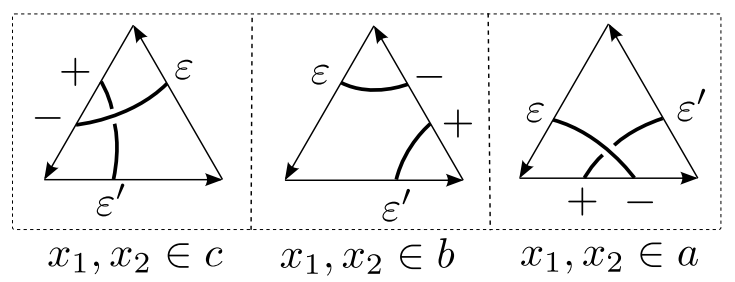

Figure 17. Case 2: From left to right we have $\alpha(-, \varepsilon) \beta\left(\varepsilon^{\prime},+\right), \alpha(\varepsilon,-) \gamma\left(+, \varepsilon^{\prime}\right)$, and $\beta(-\varepsilon) \gamma\left(\varepsilon^{\prime},+\right)$

Subcase 2(ii): $u, v \in b$. Then $\vartheta_{i} \vartheta_{j}=\alpha(\varepsilon,-) \gamma\left(+, \varepsilon^{\prime}\right)$, the left hand side of (43), see Figure 17. But we might not have $j=i+1$. We only have $i=k_{1}$ and $j=k_{1}+k_{2}+1$, so that $\bar{\vartheta}_{i}$ is the last $\alpha$ and $\bar{\vartheta}_{j}$ is the first $\gamma$ in the product $\theta^{\mathbf{k}}=\alpha^{k_{1}} \beta^{k_{2}} \gamma^{k_{3}}$. However, we can bring $\bar{\vartheta}_{i}$ next to $\bar{\vartheta}_{j}$ using (49): $\operatorname{In} \operatorname{Gr}(A)$ we have

$$
\left(\theta^{\mathbf{k}}, s\right)=\left(\alpha^{k_{1}} \beta^{k_{2}} \gamma^{k_{3}}, s\right) \doteq\left(\alpha^{k_{1}-1} \beta^{k_{2}}(\alpha \gamma) \gamma^{k_{3}-1}, s\right) \doteq\left(\alpha^{k_{1}-1} \beta^{k_{2}}(\alpha \gamma) \gamma^{k_{3}-1}, s^{\prime}\right) \doteq\left(\theta^{\mathbf{k}}, s^{\prime}\right),
$$

for some state $s^{\prime}$ with $\operatorname{nd}\left(s^{\prime}\right)<\operatorname{nd}(s)$. Here the second and the last identities are (48), and the third identity follows from (50).

Thus, in all cases we always have (51). An induction shows that for all $\left(\theta^{\mathbf{k}}, s\right) \in \vec{M}_{m}$,

$$
\left(\theta^{\mathbf{k}}, s\right) \doteq\left(\theta^{\mathbf{k}}, s^{\uparrow}\right) \quad \text { in } \operatorname{Gr}(A)
$$

This shows $\vec{M}_{m}^{\uparrow}$ also spans $\operatorname{Gr}_{m}(A)$.

Step 5. Since $\operatorname{Gr}(\omega)\left(\vec{M}_{m}^{\uparrow}\right)=B_{m}$, which is an $\mathcal{R}$-basis of $\operatorname{Gr}_{m}\left(\mathscr{S}_{\mathrm{s}}(\mathfrak{T})\right), \vec{M}_{m}^{\uparrow}$ is $\mathcal{R}$-linearly independent. Thus, $\vec{M}_{m}^{\uparrow}$ is an $\mathcal{R}$-basis of $\operatorname{Gr}_{m}(A)$, and $\operatorname{Gr}(\omega): \operatorname{Gr}_{m}(A) \rightarrow \operatorname{Gr}_{m}\left(\mathscr{S}_{\mathrm{s}}(\mathfrak{T})\right)$ is an isomorphism. It follows that $\omega: A \rightarrow \mathscr{S}_{\mathrm{s}}(\mathfrak{B})$ is an isomorphism.

The following lemma is used in the proof of Theorem 4.6, and we use notations there.

Lemma 4.7. Suppose $D$ is a stated $\partial \mathfrak{T}$-tangle diagram with $\partial D=\partial(\mathbf{k})$ and each component of $D$ is an arc. Let $s \in \mathrm{St}(\mathbf{k})$ be the state of $D$.

(a) If $D$ contains a returning arc, i.e. an arc whose two ends are in one edge of $\mathfrak{T}$, then, as an element in $\mathscr{S}_{\mathbf{s}}(\mathfrak{T}), D \in F_{|\mathbf{k}|-1}$.

(b) If $D$ has no returning ars, then, as elements in $\mathscr{S}_{\mathrm{s}}(\mathfrak{T})$,

$$
D=\theta(\mathbf{k}, s) \quad\left(\bmod F_{|\mathbf{k}|-1}\right) .
$$

Proof. (a) The proof of Lemma 4.3(a) works also for this case.

(b) If $D$ has no double point, then $D=\theta(\mathbf{k}, s)$. Suppose $D$ has a double point. Of the

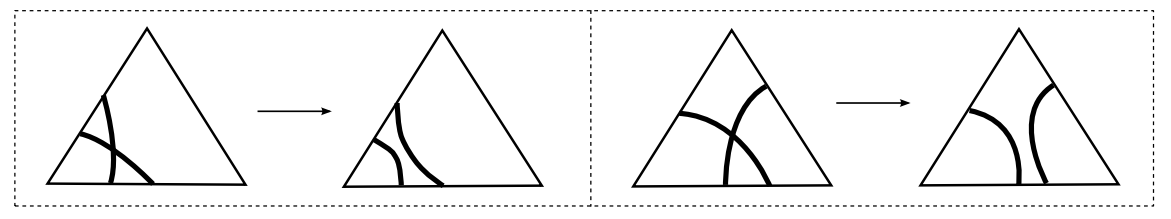

Figure 18. There are two types of double points (up to isotopies and rotations); in each case the only resolution without returning arc is drawn. Other resolutions have returning arcs 
two resolutions of the double point, exactly one does not have a returning arc; see Figure 18 . By the skein relation, part (a), and induction, we have $D=\theta(\mathbf{k}, s)\left(\bmod F_{|\mathbf{k}|-1}\right)$.

Proposition 4.8. Suppose $\mathcal{R}$ is a domain. Then $\mathscr{S}_{\mathrm{S}}(\mathfrak{T})$ is a domain. More over, if $\tilde{\mathcal{F}}$ is a collection of ideal triangles, then $\bigotimes_{\mathfrak{T} \in \tilde{\mathcal{F}}} \mathscr{S}_{\mathrm{S}}(\mathfrak{T})$ is a domain.

Proof. For $\mathbf{k}=\left(k_{1}, k_{2}, k_{3}, k_{a}, k_{b}, k_{c}\right) \in I$, where

$$
I:=\left\{\left(k_{1}, k_{2}, k_{3}, k_{a}, k_{b}, k_{c}\right) \in \mathbb{N}^{6} \mid k_{a} \leq k_{2}+k_{3}, k_{b} \leq k_{1}+k_{3}, k_{c} \leq k_{1}+k_{2}\right\},
$$

define $b_{\mathbf{k}}=\left(\alpha^{k_{1}} \beta^{k_{2}} \gamma^{k_{3}}, s\right)$, where $s \in \mathrm{St}^{\uparrow}(k)$ is the only increasing state such that there are $k_{a}$ pluses on edge $a, k_{b}$ pluses on edge $b$, and $k_{c}$ pluses on edge $c$. Then $\left\{b_{\mathbf{k}} \mid \mathbf{k} \in I\right\}$ is an $\mathcal{R}$-basis of $\operatorname{Gr}\left(\mathscr{S}_{\mathrm{s}}(\mathfrak{T})\right)$. We order $I$ using the lexicographic order. Lemma 4.7 shows that

$$
z(\mathbf{k}) z\left(\mathbf{k}^{\prime}\right) \doteq z\left(\mathbf{k}+\mathbf{k}^{\prime}\right) \quad \text { in } \operatorname{Gr}\left(\mathscr{S}_{\mathrm{s}}(\mathfrak{T})\right)
$$

Lemma 4.5 shows that $\operatorname{Gr}\left(\mathscr{S}_{\mathrm{S}}(\mathfrak{T})\right)$ is a domain. Hence $\mathscr{S}_{\mathrm{s}}(\mathfrak{T})$ is a domain.

By combining the above basis of $\mathscr{S}_{\mathbf{s}}(\mathfrak{T})$, with the lexicographic order, we get a compatibly ordered basis of $\bigotimes_{\mathfrak{T} \in \tilde{\mathcal{F}}} \mathscr{S}_{\mathrm{S}}(\mathfrak{T})$. Hence $\bigotimes_{\mathfrak{T} \in \tilde{\mathcal{F}}} \mathscr{S}_{\mathrm{S}}(\mathfrak{T})$ is a domain.

\subsection{Zero-divisor. Proof of Theorem 2.}

Proof. If $\partial \mathfrak{S}$ is a closed manifold, then $\mathscr{S}_{\mathfrak{s}}(\mathfrak{S})=\dot{\mathscr{S}}(\mathfrak{S})$, and the result is well-known and was proved in [PS2]. Assume $\partial \mathfrak{S} \neq \emptyset$. There are only a few simple cases when $\mathfrak{S}$ is not triangulable, listed in (i)-(iv) below. In each case, $\mathfrak{S}=\overline{\mathfrak{S}} \backslash \mathcal{P}$.

(i) $\overline{\mathfrak{S}}=S^{2},|\mathcal{P}|=1$. Then $\mathscr{S}_{\mathrm{s}}(\mathfrak{S})=\dot{\mathscr{S}}(\mathfrak{S})=\mathcal{R}$, which is domain.

(ii) $\overline{\mathfrak{S}}=S^{2},|\mathcal{P}|=2$. Then $\mathscr{S}_{\mathfrak{S}}(\mathfrak{S})=\dot{\mathscr{S}}(\mathfrak{S})=\mathcal{R}[x]$, which is a domain. Here $x$ is is only non-trivial simple loop in $\mathfrak{S}$.

(iii) $\overline{\mathfrak{S}}$ is a disk and $|\mathcal{P}|=1$. Then $\mathscr{S}_{\mathfrak{S}}(\mathfrak{S})=\mathcal{R}$, a domain.

(iv) $\mathfrak{S}$ is an ideal bigon. Then $\mathscr{S}_{\mathbf{S}}(\mathfrak{S})$ is a domain by Proposition 4.4.

Now suppose $\mathfrak{S}$ has an ideal triangulation. By the triangular decomposition, $\mathscr{S}_{\mathfrak{S}}(\mathfrak{S})$ embeds into $\bigotimes_{\mathfrak{T} \in \tilde{\mathcal{F}}} \mathscr{S}_{\mathrm{S}}(\mathfrak{T})$, which is a domain by Proposition 4.8. It follows that $\mathscr{S}_{\mathrm{S}}(\mathfrak{S})$ is a domain.

Remark 4.9. Besides the case when $\partial \mathfrak{S}$ is a closed manifold, when $\partial \mathfrak{S}=\emptyset$, we also have $\mathscr{S}_{\mathrm{s}}(\mathfrak{S})=\dot{\mathscr{S}}(\mathfrak{S})$, and the fact that $\dot{\mathscr{S}}(\mathfrak{S})$ is a domain was known before, see [PS2, BW1, CM]. Our proof in these special cases is different from those in [PS2, BW1, CM]. Later we show that the Muller skein algebra embeds into $\mathscr{S}_{\mathbf{S}}(\mathfrak{S})$, hence it is also a domain, a fact proven by Muller before using another method [Mu].

Remark 4.10. Suppose $\mathcal{R}=\mathbb{C}, q=-1$, and $\mathfrak{S}$ is a triangulated punctured bordered surfaced with a triangulation $\Delta$. Then $\mathscr{S}_{\mathbb{S}}(\mathfrak{S})$ is canonically isomorphic to the ring of regular functions on the $S L_{2}(\mathbb{C})$-character variety of $\pi_{1}(\mathfrak{S})$. The triangular decomosition shows there is a natural embedding of $\mathscr{S}_{\mathrm{S}}(\mathfrak{S})$ into $\bigotimes_{\mathfrak{T} \in \tilde{\mathcal{F}}} \mathscr{S}_{\mathrm{S}}(\mathfrak{T})$, which is non-commutative. Even in this case of $q=-1$, the ring $\mathscr{S}_{\mathbf{s}}(\mathfrak{T})$ and the triangular decomposition seem new. 


\section{Chekhov-Fock algebra And quantum trace}

5.1. Chekhov-Fock triangle algebra, Weyl normalization. Suppose $\mathfrak{T}$ is an ideal triangle with boundary edges $a, b, c$ and $\operatorname{arcs} \alpha, \beta, \gamma$ as in Figure 11. Define $\mathcal{Y}(\mathfrak{T})$ to be the $\mathcal{R}$-algebra

$$
\mathcal{Y}(\mathfrak{T})=\mathcal{R}\left\langle y_{a}^{ \pm 1}, y_{b}^{ \pm 1}, y_{c}^{ \pm 1}\right\rangle /\left(y_{a} y_{b}=q y_{b} y_{a}, y_{b} y_{c}=q y_{c} y_{b}, y_{c} y_{a}=q y_{c} y_{a}\right)
$$

Then $\mathcal{Y}(\mathfrak{T})$ belongs to a type of algebras called quantum tori, see eg. [Le, Section 2].

Suppose $x, y$ are elements of an $\mathcal{R}$-algebra such that $x y=q^{k} y x$, where $k \in \mathbb{Z}$. Define the Weyl normalization of $x y$ by

$$
[x y]:=q^{-k / 2} x y=q^{k / 2} y x .
$$

The advantage is that $[x y]=[y x]$. For example, for $b, c \in \mathcal{Y}(\mathfrak{T})$ and $\varepsilon, \varepsilon^{\prime} \in\{ \pm 1\}$, we have

$$
\left[\left(y_{c}\right)^{\varepsilon}\left(y_{b}\right)^{\varepsilon^{\prime}}\right]=q^{\varepsilon \varepsilon^{\prime} / 2}\left(y_{c}\right)^{\varepsilon}\left(y_{b}\right)^{\varepsilon^{\prime}} \text {. }
$$

The rotation $\tau: \mathfrak{T} \rightarrow \mathfrak{T}$, which gives the cyclic permutations $\alpha \rightarrow \beta \rightarrow \gamma \rightarrow \alpha$ and $a \rightarrow b \rightarrow c \rightarrow a$, induces algebra automorphisms of the algebras $\mathscr{S}_{\mathrm{S}}(\mathfrak{T})$ and $\mathcal{Y}(\mathfrak{T})$.

Proposition 5.1. There exists a unique $\mathcal{R}$-algebra homomorphism $\phi: \mathscr{S}_{\mathrm{s}}(\mathfrak{T}) \rightarrow \mathcal{Y}(\mathfrak{T})$ which is $\tau$-equivariant and satisfies

$$
\phi\left(\alpha\left(\varepsilon \varepsilon^{\prime}\right)\right)= \begin{cases}0 & \text { if } \varepsilon=-, \varepsilon^{\prime}=+ \\ {\left[c^{\varepsilon} b^{\varepsilon^{\prime}}\right]} & \text { otherwise. }\end{cases}
$$

Proof. The proof follows from an easy checking that the definition (55) respects all the defining relations of $\mathscr{S}_{\mathrm{s}}(\mathfrak{T})$ described in Proposition 4.6.

5.2. Quantum trace. Let $\Delta$ be a triangulation of a punctured bordered surface $\mathfrak{S}$ and $\tilde{\mathcal{F}}=\tilde{\mathcal{F}}(\Delta)$ be the collection of disjoint ideal triangles obtained by splitting $\mathfrak{S}$ along the edges of $\Delta$, see Section 3.3. Let $\mathcal{E}$ be the set of all edges of $\Delta$, and $\tilde{\mathcal{E}}$ be the set of all edges of all triangles in $\tilde{\mathcal{F}}$.

Using the triangular decomposition (32) and the algebra map $\phi$ of Section 5.1, define $\varkappa_{\Delta}$ as the composition

$$
\varkappa_{\Delta}: \mathscr{S}_{\mathrm{S}} \stackrel{\rho_{\Delta}}{\longrightarrow} \bigotimes_{\mathfrak{T} \in \tilde{\mathcal{F}}(\Delta)} \mathscr{S}_{\mathrm{S}}(\mathfrak{T}) \stackrel{\otimes \phi}{\longrightarrow} \mathcal{Y}(\tilde{\mathcal{F}}):=\bigotimes_{\mathfrak{T} \in \tilde{\mathcal{F}}(\Delta)} \mathcal{Y}(\mathfrak{T})
$$

Bonahon and Wong [BW1] constructed an algebra homomorphism (quantum trace map)

$$
\operatorname{Tr}_{\Delta}: \widehat{\mathscr{S}_{s}}(\mathfrak{S}) \rightarrow \mathcal{Y}(\Delta)
$$

where $\mathcal{Y}(\Delta)$, a version of the Chekhov-Fock algebra, is an $\mathcal{R}$-subalgebra of $\mathcal{Y}(\tilde{\mathcal{F}})$. For now, we consider $\operatorname{Tr}_{\Delta}$ as a map with target $\mathcal{Y}(\tilde{\mathcal{F}})$. We recall the definition of $\mathcal{Y}(\Delta)$ in Section 5.3 ,

Theorem 5.2. If $\Delta$ is an ideal triangulation of a punctured bordered surface $\mathfrak{S}$, then the composition $\widehat{\varkappa_{\Delta}}: \widehat{\mathscr{S}}_{\mathrm{S}}(\mathfrak{S}) \rightarrow \mathscr{S}_{\mathrm{S}}(\mathfrak{S}) \stackrel{\varkappa_{\Delta}}{\longrightarrow} \mathcal{Y}(\tilde{\mathcal{F}})$ is equal to the quantum trace map of Bonahon and Wong. 
Proof. (i) The case $\mathfrak{S}=\mathfrak{T}$, an ideal triangle with notations of Figure 11, In this case $\widehat{\mathscr{S}_{s}}(\mathfrak{T})$ is generated by $\alpha\left(\varepsilon, \varepsilon^{\prime}\right)$, the $\operatorname{arcs} \delta\left(\varepsilon, \varepsilon^{\prime}\right)$, and their images under $\tau, \tau^{2}$. Here $\delta\left(\varepsilon, \varepsilon^{\prime}\right)$ is a returning arc with states $\varepsilon, \varepsilon^{\prime}$. For each of these generators, the image of $\operatorname{Tr}_{\Delta}$ described in [BW1, Theorem 11] is exactly the image of $\varkappa_{\Delta}$ given by (17) and (55). Hence $\widehat{\varkappa_{\Delta}}=\operatorname{Tr}_{\Delta}$.

(ii) Now return to the case of general punctured surfaces. Suppose $e_{1}, e_{2}$ are edges of $\mathfrak{S}$ and $\mathfrak{S}^{\prime}=\mathfrak{S} /\left(e_{1}=e_{2}\right)$. Then $\mathfrak{S}^{\prime}$ inherits a triangulation $\Delta^{\prime}$ from $\Delta$, where the set $\mathcal{E}^{\prime}$ of edges of $\Delta^{\prime}$ is the same as $\mathcal{E}$, except that the two edges $e_{1}, e_{2}$ of $\mathcal{E}$ are glued together, giving an edge $e$ of $\mathcal{E}^{\prime}$. Both $\mathfrak{S}$ and $\mathfrak{S}^{\prime}$ are obtained from the same collection $\tilde{\mathcal{F}}$ of ideal triangles by identifications of edges, with $\mathfrak{S}^{\prime}$ having one more identification.

By [BW1, Theorem 11], $\operatorname{Tr}_{\Delta}$ is uniquely characterized by its values for ideal triangles and the the following condition: For any such pair $\mathfrak{S}, \mathfrak{S}^{\prime}$ and any stated $\partial \mathfrak{S}^{\prime}$-tangle $\alpha$ vertically transversal to $e$,

$$
\operatorname{tr}_{\Delta^{\prime}}(\alpha)=\sum_{\beta} \operatorname{Tr}_{\Delta}(\beta)
$$

where $\beta$ runs the set of all lifts of $\alpha$, see Section 3.1. The sequence of maps

$$
\mathscr{S}_{\mathrm{S}}\left(\mathfrak{S}^{\prime}\right) \stackrel{\rho}{\longrightarrow} \mathscr{S}_{\mathrm{S}}(\mathfrak{S}) \stackrel{\rho_{\Delta}}{\longrightarrow} \bigotimes_{\mathfrak{T} \in \tilde{\mathcal{F}}(\Delta)} \mathscr{S}_{\mathrm{S}}(\mathfrak{T}) \stackrel{\otimes \phi}{\longrightarrow} \mathcal{Y}(\tilde{\mathcal{F}})
$$

shows that $\varkappa_{\Delta^{\prime}}=\varkappa_{\Delta} \circ \rho$. Hence, from the definition of $\rho$, we have

$$
\widehat{\varkappa_{\Delta^{\prime}}}(\alpha)=\sum_{\beta} \widehat{\varkappa_{\Delta}}(\beta)
$$

i.e. $\widehat{\varkappa_{\Delta}}$ also satisfies the above condition (56). This proves $\widehat{\varkappa_{\Delta}}=\operatorname{Tr}_{\Delta}$.

We have seen that the triangular decomposition (32) gives a simple proof of the existence of the quantum trace map of Bonahon and Wong [BW1]. Because there is no analog of $\rho$ relating $\widehat{\mathscr{S}_{s}}\left(\mathfrak{S}^{\prime}\right)$ and $\widehat{\mathscr{S}_{s}}(\mathfrak{S})$, in $[\mathrm{BW}$ ] the quantum trace map has to be defined directly on $\widehat{\mathscr{S}_{s}}(\mathfrak{S})$, and the proof of well-definedness involves difficult calculations. For yet another proof of the existence of the quantum trace map, the reader can consult [Le].

5.3. Chekhov-Fock algebra. We continue with the notation of the previous subsection. For each $\mathfrak{T} \in \tilde{\mathcal{F}}$ we consider $\mathcal{Y}(\mathfrak{T})$ as a subalgebra of $\mathcal{Y}(\tilde{\mathcal{F}})=\bigotimes_{\mathfrak{T} \in \tilde{\mathcal{F}}(\Delta)} \mathcal{Y}(\mathfrak{T})$ under the natural embedding. Then $\mathcal{Y}(\mathfrak{T})$ is the $\mathcal{R}$-algebra generated by $y_{e}^{ \pm 1}$ with $e \in \tilde{\mathcal{E}}$, subject to the relation $y_{e_{1}} y_{e_{2}}=y_{e_{2}} y_{e_{1}}$ if $e_{1}$ and $e_{2}$ are edges of different triangles, and $y_{e_{1}} y_{e_{2}}=q y_{e_{2}} y_{e_{1}}$ if $e_{2}$ and $e_{1}$ are edges of a triangle and $e_{2}$ follows $e_{1}$ in counterclockwise order.

Under the natural projection pr $: \tilde{\mathcal{E}} \rightarrow \mathcal{E}=\mathcal{E}(\Delta)$, an edge $e \in \mathcal{E}$ has one or two pre-images in $\tilde{\mathcal{E}}$; each is called a lift of $e$. For each $e \in \mathcal{E}$ define $y_{a} \in \mathcal{Y}(\mathfrak{T})$ by

- If $e$ has a unique lift $e^{\prime} \in \tilde{\mathcal{E}}$, then $y_{e}=y_{e^{\prime}}$.

- If $e$ has two lifts $e^{\prime}, e^{\prime \prime} \in \tilde{\mathcal{E}}$, then $y_{e}=\left[y_{e^{\prime}} y_{e^{\prime \prime}}\right]$.

Let $\mathcal{Y}(\Delta)$ be the $\mathcal{R}$-subalgebra of $\mathcal{Y}(\mathfrak{T})$ generated by $y_{a}^{ \pm 1}, a \in \mathcal{E}$. Then $\mathcal{Y}(\Delta)$ is a version of the (multiplicative) Chekhov-Fock algebra of $\mathfrak{S}$ associated with the triangulation $\Delta$. In [BW1, it proved that the image of $\operatorname{Tr}_{\Delta}$ is in $\mathcal{Y}(\Delta)$, which can also be proved easily from the definition of $\varkappa_{\Delta}$. 


\section{Relation with the skein algebra of Muller}

6.1. Skein algebra of marked surface. Recall that a marked surface $(\overline{\mathfrak{S}}, \mathcal{P})$ consists of a compact oriented 2-dimensional manifold $\overline{\mathfrak{S}}$ with (possibly empty) boundary $\partial \overline{\mathfrak{S}}$ and a finite set $\mathcal{P} \subset \partial \overline{\mathfrak{S}}$. We recall the definition of the Muller skein algebra [Mu], following [Le].

Let $\mathfrak{S}=\overline{\mathfrak{S}} \backslash\left(\mathcal{P} \cup \partial^{\prime}(\overline{\mathfrak{S}})\right)$, where $\partial^{\prime}(\overline{\mathfrak{S}})$ is the union of all connected components of $\partial \overline{\mathfrak{S}}$ which do not intersect $\mathcal{P}$. Then $\mathfrak{S}$ is a punctured marked surface.

$A \mathcal{P}$-tangle $\alpha$ is defined just like a $\partial \mathfrak{S}$-tangle, only with $\partial(\alpha) \subset \mathcal{P} \times(0,1)$. More precisely, a $\mathcal{P}$-tangle $\alpha$ is a compact, framed, properly embedded 1-dimensional non-oriented smooth submanifold $\alpha$ of $\overline{\mathfrak{S}} \times(0,1)$ such that $\partial(\alpha) \subset \mathcal{P} \times(0,1)$ and at every boundary point of $\alpha$ the framing is vertical. Two $\mathcal{P}$-tangles are isotopic if they are isotopic through the class of $\mathcal{P}$-tangles. Define $\mathscr{S}^{\text {Muller }}(\overline{\mathfrak{S}}, \mathcal{P})$ to be the $\mathcal{R}$-module freely spanned by isotopy classes of $\mathcal{P}$-tangles modulo the skein relation (10), the trivial loop relation (11), and the new trivial arc relation (see Figure 19).

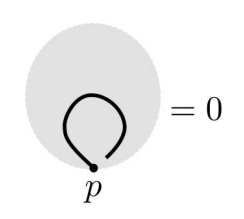

Figure 19. Trivial arc relation

More precisely, the trivial arc relation says $\alpha=0$ for any $\mathcal{P}$-tangle $\alpha$ of the form $\alpha=\alpha^{\prime} \sqcup a$, where $a \subset \overline{\mathfrak{S}} \times(0,1) \backslash \alpha^{\prime}$ is an arc with two end points in $p \times(0,1)$ for some $p \in \mathcal{P}$, such that $a$ and the part of $p \times(0,1)$ between the two end points of $a$ co-bound a disk in $\overline{\mathfrak{S}} \times(0,1) \backslash \alpha^{\prime}$.

As usual, the product of two $\mathcal{P}$-tangles is obtained by stacking the first on top of the second. With this product, $\mathscr{S}^{\text {Muller }}(\overline{\mathfrak{S}}, \mathcal{P})$ is an $\mathcal{R}$-algebra.

Let $\mathscr{S}_{s,+}(\mathfrak{S}) \subset \mathscr{S}_{\mathfrak{S}}(\mathfrak{S})$ be the $\mathcal{R}$-submodule spanned by stated $\partial \mathfrak{S}$-tangles whose states are all + . For an $\mathcal{P}$-tangle $\alpha$ define a stated $\partial \mathfrak{S}$-tangle $\tilde{\Omega}(\alpha)$ by moving all the boundary points of $\alpha$ slightly to the left (i.e. along the positive direction of $\partial \overline{\mathfrak{S}}$ ), keeping the same height, and equipping $\tilde{\Omega}(\alpha)$ with state + at every boundary points. Relations (10)-(12) show that $\tilde{\Omega}$ descends to a well-define $\mathcal{R}$-linear map

$$
\Omega: \mathscr{S}^{\text {Muller }}(\overline{\mathfrak{S}}, \mathcal{P}) \rightarrow \mathscr{S}_{s,+}(\mathfrak{S})
$$

Proposition 6.1. The map $\Omega$ is an $\mathcal{R}$-algebra isomorphism.

Proof. It is easy to see that $\mathscr{S}_{s,+}(\mathfrak{S})$ is the $\mathcal{R}$-module freely spanned by $\partial \mathfrak{S}$-tangles with + states, subject to those relations from (10)-(13) which involve only + states; namely relations (10), (11), and the middle relation of (12). Since $\tilde{\Omega}$ maps the set of isotopy classes of $\mathcal{P}$-tangles isomorphically onto the set of isotopy classes of + stated $\partial \mathfrak{S}$-tangles, and maps the defining relations of $\mathscr{S}^{\text {Muller }}(\overline{\mathfrak{S}}, \mathcal{P})$ onto the defining relations of $\mathscr{S}_{s,+}(\mathfrak{S})$, it induces an isomorphism $\Omega: \mathscr{S}^{\text {Muller }}(\overline{\mathfrak{S}}, \mathcal{P}) \rightarrow \mathscr{S}_{s,+}(\mathfrak{S})$.

Alternatively, the $\mathcal{R}$-basis of $\mathscr{S}^{\text {Muller }}(\overline{\mathfrak{S}}, \mathcal{P})$, given explicitly in [Mu. Lemma 4.1], is mapped by $\Omega$ to the $\mathcal{R}$-basis of $\mathscr{S}_{s,+}(\mathfrak{S})$ given in Theorem 2.8 , with all + states. This shows $\Omega$ is an isomorphism. 


\subsection{Proof of Theorem 4.}

Proof. Recall that we define the quantum trace map on $\mathscr{S}^{\text {Muller }}(\overline{\mathfrak{S}}, \mathcal{P})$

$$
\bar{\varkappa}_{\Delta}: \mathscr{S}^{\text {Muller }}(\overline{\mathfrak{S}}, \mathcal{P}) \stackrel{\Omega}{\longrightarrow} \mathscr{S}_{s,+}(\mathfrak{S}) \stackrel{\varkappa_{\Delta}}{\longrightarrow} \mathcal{Y}(\Delta) \text {. }
$$

Number the set $\mathcal{E}$ of edges of $\Delta$ so that $\mathcal{E}=\left\{e_{1}, \ldots, e_{n}\right\}$. For $\mathbf{k}=\left(k_{1}, \ldots, k_{n}\right) \in \mathbb{Z}^{n}$ let

$$
y^{\mathbf{k}}:=\left(y_{e_{1}}\right)^{k_{1}}\left(y_{e_{2}}\right)^{k_{2}} \ldots\left(y_{e_{n}}\right)^{k_{n}} \in \mathcal{Y}(\Delta)
$$

The set $\left\{y^{\mathbf{k}} \mid \mathbf{k} \in \mathbb{Z}\right\}$ is an $\mathcal{R}$-basis of $\mathcal{Y}(\Delta)$. We order all $y^{\mathbf{k}}$ using the lexicographic order of $\mathbf{k} \in \mathbb{Z}^{n}$, and use this order to define the leading term $\operatorname{lt}(x)$ of any $0 \neq x \in \mathcal{Y}(\Delta)$.

Let $\tilde{\mathfrak{S}}=\bigsqcup_{\mathfrak{T} \in \tilde{\mathcal{F}}} \mathfrak{T}$, and pr $: \tilde{\mathfrak{S}} \rightarrow \mathfrak{S}$ be the natural projection. Suppose $D$ is a simple dS-tangle diagram which is $\Delta$-normal, i.e. it is $e$-normal for all edge $e$ of $\Delta$ (see Proof of Theorem 3.1(b)). Then $\tilde{D}:=\operatorname{pr}^{-1}(D)$ is a $\partial \tilde{\mathfrak{S}}$-tangle diagram consisting of non-returning arcs in ideal triangles in $\tilde{\mathcal{F}}$. Define $\mathbf{k}_{D}=\left(k_{D, 1}, \ldots, k_{D, n}\right) \in \mathbb{Z}^{n}$, where $k_{D, i}=\left|D \cap e_{i}\right|$. Then $\mathbf{k}_{D}$ totaly determines the isotopy class of $D$. Every simple $\partial \mathfrak{S}$-tangle diagram is isotopic to a $\Delta$-normal one.

An edge $e$ of $\Delta$ is interior if it is not a boundary edge of $\mathfrak{S}$. Let $\stackrel{\circ}{E}$ be the union of all the interior edges of $\Delta$. Fix an orientation of $\stackrel{\circ}{E}$ and provide the boundary edges of $\mathfrak{S}$ with the positive orientation. Lift these orientations to edges of $\tilde{\mathcal{E}}$, and get an orientation $\mathfrak{o}$ of $\partial \tilde{\mathfrak{S}}$. Suppose $D$ is a positively ordered, $\Delta$-normal, simple $\partial \mathfrak{S}$-tangle diagram, with + states. For every map $r: D \cap \stackrel{\circ}{E} \rightarrow\{ \pm\}$, let $\tilde{D}(r)$ be $\tilde{D}$ with $\mathfrak{o}$-order, and states defined by the lift of $r$ on $E^{\circ}$ and + on all other edges. From the definition, we have the following state sum

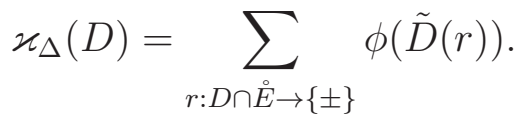

Let $r_{+}$be the map $r_{+}: D \cap \stackrel{\circ}{E} \rightarrow\{+\}$. Since $\tilde{D}\left(r_{+}\right)$consists of non-returning arcs with + states, (55) and Lemma 4.7 show that $\phi\left(\tilde{D}\left(r_{+}\right)\right)$is non-zero and

$$
\operatorname{lt}\left(\phi\left(\tilde{D}\left(r_{+}\right)\right)\right) \doteq y^{\mathbf{k}_{D}} .
$$

More over (55) and Lemma 4.7 show that the leading term of any $\phi(\tilde{D}(r))$, with $r \neq r_{+}$, is smaller than that of $\phi\left(\tilde{D}\left(r_{+}\right)\right)$. Hence

$$
\operatorname{lt}\left(\varkappa_{\Delta}(D)\right) \doteq y^{\mathbf{k}_{D}} .
$$

From here it is easy to see that the image under $\varkappa_{\Delta}$ of a non-trivial linear combination of + stated, positively ordered, $\Delta$-normal, simple $\partial \mathfrak{S}$-tangle diagrams is non-zero, which proves that $\bar{\varkappa}_{\Delta}$ is injective.

\section{REFERENCES}

[BHMV] C. Blanchet, N. Habegger, G. Masbaum, and P. Vogel, Three-manifold invariants derived from the Kauffman bracket, Topology 31 (1992), no. 4, 685-699.

[Bul] D. Bullock, Rings of $S l_{2}(\mathbb{C})$-characters and the Kauffman bracket skein module, Comment. Math. Helv. 72 (1997), no. 4, 521-542.

$[\mathrm{BFK}]$ D. Bullock, C. Frohman, and J. Kania-Bartoszynska, Understanding the Kauffman bracket skein module, J. Knot Theory Ramifications 8 (1999), no. 3, 265-277. 
[BP] D. Bullock and J. Przytycki, Multiplicative structure of Kauffman bracket skein module quantizations, Proc. Amer. Math. Soc. 128 (2000), no. 3, 923-931.

[BW1] F. Bonahon and H. Wong, Quantum traces for representations of surface groups in SL2(C), Geom. Topol. 15 (2011), no. 3, 1569-1615.

[BW2] F. Bonahon and H. Wong, Representations of the Kauffman skein algebra I: invariants and miraculous cancellations, preprint arXiv:1206.1638.

[CF1] L. Chekhov and V. Fock, Quantum Teichmüller spaces (Russian) Teoret. Mat. Fiz. 120 (1999), no. 3, 511-528; translation in Theoret. and Math. Phys. 120 (1999), no. 3, 1245-1259.

[CF2] L. O. Chekhov and V. V. Fock, Observables in 3D gravity and geodesic algebras, in: Quantum groups and integrable systems (Prague, 2000), Czechoslovak J. Phys. 50 (2000), 1201-1208.

[CM] L. Charles and J. Marché, Multicurves and regular functions on the representation variety of a surface in SU(2), Comment. Math. Helv. 87 (2012), 409-431.

[Hi] C. Hiatt, Quantum traces in quantum Teichmüller theory, Algebr. Geom. Topol. 10 (2010), 12451283.

[Fo] V. V. Fock, Dual Teichmüller spaces, unpublished preprint, 1997, arXiv:Math/dg-ga/9702018.

[Jo] V. Jones, Polynomial invariants of knots via von Neumann algebras, Bull. Amer. Math. Soc., 12 (1985), 103-111.

[Kau] L. Kauffman, States models and the Jones polynomial, Topology, 26 (1987), 395-407.

[Kas] R. Kashaev, Quantization of Teichmüller spaces and the quantum dilogarithm, Lett. Math. Phys. 43 (1998), no. 2, 105-115.

[Le] T. T. Q. Lê, The colored Jones polynomial and the AJ conjecture, in "Lectures on quantum topology in dimension three", Panoramas et synthèses series, Publications of Soc. Math. France. In press.

[Le] T. T. Q. Lê, Quantum Teichmuller spaces and quantum trace map, preprint arXiv:1511.06054, 2015.

[LZ] T. T. Q. Lê and X. Zhang Character varieties, A-polynomials, and the AJ Conjecture, preprint arXiv:1509.03277, 2015. To appear in Algebr. Geom. Topol.

[Liu] X. Liu, The quantum Teichmüller space as a noncommutative algebraic object, J. Knot Theory Ramifications 18 (2009), 705-726.

[Mu] G. Muller, Skein algebras and cluster algebras of marked surfaces, Preprint arXiv:1204.0020, 2012. To appear in Quantum Topology.

[Pr] J. Przytycki, Fundamentals of Kauffman bracket skein modules, Kobe J. Math. 16 (1999) 45-66.

[PS1] J. Przytycki and A. Sikora, On the skein algebras and $S l_{2}(\mathbb{C})$-character varieties, Topology 39 (2000), $115-148$.

[PS2] J. Przytycki and A. Sikora, Skein algebras of surfaces, preprint arXiv:1602.07402, 2016.

[SW] A. Sikora and B.W. Westbury, Confluence Theory for Graphs, Alg. Geom. Topol. 7 (2007) 439-478.

[Tu] V. Turaev, Skein quantization of Poisson algebras of loops on surfaces, Ann. Sci. Sc. Norm. Sup. (4) 24 (1991), no. 6, 635-704.

[Vo] A. A. Voronov, Lecture notes. Available at http://www.math.umn.edu/ voronov/18.276/lec5/lec5.pdf

School of Mathematics, 686 Cherry Street, Georgia Tech, Atlanta, GA 30332, USA

E-mail address: letu@math.gatech.edu 